\title{
The use of Design of Experiments for steady-state and transient inverse melanoma detection problems
}

\author{
J. Iljaž ${ }^{* 1}$, L. C. Wrobel ${ }^{2}$, M. Hriberšek ${ }^{1}$ and J. Marn ${ }^{1}$ \\ 1 - Faculty of Mechanical Engineering, University of Maribor \\ Smetanova 17, SI-2000 Maribor, Slovenia, * corresp: jurij.iljaz@um.si \\ 2 - Brunel University London, Kingston Lane, Uxbridge, UB8 3PH, United Kingdom
}

April 30, 2018

\begin{abstract}
Melanoma is one of the most fatal skin cancers; for this reason, there is a need for the development of new safe, non-invasive and efficient diagnostic techniques. Dynamic thermography is showing to be a promising technique for the early detection of skin cancers. Therefore, this paper investigates two different inverse bioheat problems using steady-state and transient skin temperature measurements. Both problems are investigated numerically to estimate how accurate blood perfusion rate, metabolic heat generation, diameter and thickness of the tumour can be estimated simultaneously under exact and noisy measurement data, based on a complex numerical model describing multilayer tissue. The inverse problems have been tested using different melanoma size, Clark II and Clark IV. The Design of Experiments (DOE) technique has been used to solve and analyse the inverse problems. A substantial number of numerical model evaluations, totalling 2, 306, 486 simulations, had to be undertaken as part of the full factorial DOE. The results show that it is always possible to obtain tumour parameters using exact static or dynamic measurement data. However, for noisy temperature data, the use of a dynamic approach showed an advantage over the steady-state one, which failed because of the very small temperature differences between the healthy skin and the tumour. The dynamic thermography can retrieve blood perfusion rate, thickness and diameter of the tumour as well as the metabolic heat generation despite the low sensitivity for low and high levels of measurement error; however, to detect melanoma lesions at an early stage, the measurement and model errors should be kept as low as possible.
\end{abstract}

Keywords: bio-heat, melanoma, inverse problem, DOE, response surface, steadystate, transient, thermography.

\section{Introduction}

Skin cancer can be generally categorized into melanoma and non-melanoma, and further divided into pigmented and non-pigmented lesions. Among all types of skin cancer, 
melanoma is the most fatal because it metastases rapidly and can spread to soft tissues like lung and liver [1]. According to Clark et el. [2] and Breslow [3], the survival rate of patients with malignant melanoma is directly correlated with its thickness or level of invasion. The deeper the invasion, the lower the survival rate. For this reason, it is important to detect skin cancer in its early stage and it is essential to develop accurate and sensitive diagnostic techniques in order to enable early detection and diagnosis.

Nowadays the most frequently used diagnostic technique is visual inspection based on the ABCDE (Asymmetry, Border, Colour, Diameter, Evolution) criteria and dermatoscopes. As it is known, the ABCDE criteria uses only qualitative guidelines for melanoma identification and can therefore produces high rates of false positives or false negative identification. To avoid the risk of missing an early stage melanoma, excisional biopsies are performed for further pathological investigation [4-7]. Therefore, new techniques for skin cancer detection are being developed like digital photography, multispectral imaging systems, confocal scanning laser microscopy (CSLM), laser Doppler perfusion imaging (LDPI) optical coherence tomography (OCT), ultrasound and magnetic resonance imaging (MRI) [8-16]. Usually, they are a compromise between certain aspects like effectiveness, accuracy, cost and invasiveness.

Thermography or thermal tomography is offering a new non-invasive diagnostic technique for medicine in different fields, based on the bioheat transfer of the observed tissue. This is nowadays possible due to the development of infrared (IR) thermal cameras, computers and numerical modeling. Bioheat transfer is mainly governed by blood perfusion and metabolic heat generation, which affect the temperature difference between the normal and abnormal tissues. There have been many studies of thermography in the field of breast cancer, skin cancer, vascular diseases, thyroid gland disease, eye diseases as well as therapeutic assessment [17-30]. Thermography can be done passively (static) or actively (dynamic). Static thermography involves recording the skin temperature under steady-state conditions, which is time consuming because the patient has to acclimatise to the environment in the temperature controlled room [31]. On the other hand, active thermography involves introducing external thermo-stimulation like heating or cooling to induce thermal contrast between the healthy and investigated tissue. Thermal contrast is the effect of different thermal and physiological properties of tissues. Based on the thermography measurement data a mathematical or numerical model can then provide some information about the tissue state under investigation. The general advantage of dynamic thermography is that it is faster than the static one and can reveal more information about the tissue or tumour under investigation [19, 20, 22, 26, 30, 32].

In order to estimate the physiological parameters of the tumour, its size and position, based on static or dynamic thermo-graphic measurements, we have to solve an inverse bioheat problem based on the appropriate numerical model of the corresponding system. In this paper, we focus on the detection of melanoma lesion based only on the skin surface temperature measurements; however, the numerical techniques could be generally applied to other similar applications like breast tumour detection.Most research work to determine the tumour characteristics in general are based on steady-state temperature measurements (static thermography) for 2D and 3D using evolutionary algorithms [33-37]. They show that if the thermal properties, blood perfusion and metabolic heat generation of the tumour and surrounding tissue are predetermined, the size and location of the tumour can be found fairly successfully even for noisy measurement data. For the case of skin tumour identification, Luna et al. [36] tried to estimate the size of the 
tumour and its blood perfusion rate for steady-state surface temperature measurements using a simulated annealing (SA) algorithm. The results are promising even with noisy measurement data for the case of two searched parameters, diameter and blood perfusion of the tumour or thickness and blood perfusion.

All the previous papers used simplified computational domains containing only two regions, healthy tissue and tumour or lesion. More complex numerical models using multilayer tissue structures have been developed by Hossain et al. [38], Bhowmik and Repaka [39] and Hatwar and Herman [30]. Most interesting is the work of Bhowmik and Repaka [39], who tried to identify several skin tumour parameters based on steadystate skin surface temperature measurements considering a 3D multilayer tissue. Their models used genetic algorithm (GA) and SA to compare the two different approaches. The results show that the solution of inverse problems is strongly dependent on the value of other parameters, which is not desirable for new diagnostic techniques. The recent work of Hatwar and Herman [30] presents the estimation of tumour radius, depth and blood perfusion rate of breast tumours based on a $2 \mathrm{D}$ numerical model including multilayer tissue, using commercially available software and the Levenberg-Marquardt optimization algorithm. Under steady-state temperature measurement, they were able to detect tumour dimension and depth and they demonstrate numerically that the blood perfusion rate cannot be simultaneously detected. For that, transient surface temperature measurements are needed, where they took only transient measurements at the middle surface point. Herein, a better reconstruction of parameters is achieved using greater surface and time temperature measurement resolution also for deeper tumour locations. Although breast cancer parameter estimation is not the topic of this paper, there are similarities between our numerical techniques and those of [30].

This paper analyzes two different inverse bioheat problems of estimating several skin tumour parameters based on skin surface temperature measurements. The first inverse problem deals with steady-state temperature measurements (static thermography), while the second uses transient measurements (dynamic thermography) as proposed by Çetingül and Herman [26]. The number of parameters to be determined is four: diameter, thickness, blood perfusion rate and metabolic heat generation of the melanoma, from which the thickness and blood perfusion are the most important factors in reflecting the stage and progression of the skin tumour. The numerical model to estimate these parameters is based on the work of Çetingül and Herman [26], Bhowmik and Repaka [39] and Cheng and Herman [32] using a 2D computational domain and considering a multilayer tissue structure that better reflects the real problem.

The non-linear numerical model for solution of the direct problem is based on a subdomain Boundary Element Method (BEM) solver presented in our previous paper [40], where it is shown that it can solve direct bioheat problems accurately and efficiently, which is desirable when solving inverse problems. The optimisation technique for solving inverse bioheat problems presented in this paper is the full factorial Design of Experiments (DOE) not using the surrogate model (SM). The reason for choosing the full factorial DOE is that it is possible to explore the complete design space, and to analyse and compare the differences between static and dynamic thermography. The DOE allows to plot the objective function (response surface) and to estimate the sensitivity of each parameter, which helps in analysing which parameters can be estimated easily and which parameters cannot be estimated at all. The DOE for both inverse problems required 2,306,486 evaluations of the numerical model, which is substantial, time consuming and impractical 
for clinical implementation. However, it is valuable in this stage of investigation due to the design space and problem exploration, which could not be done using other algorithms. The paper shows inverse problem analyses for steady-state and transient measurement approaches based on two melanoma sizes (Clark II and Clark IV) under exact as well as noisy measurement data.

The novelty of this paper can be found in the solution of steady-state and transient inverse bioheat problems to estimate four important skin tumour parameters using complex numerical models. Another novelty is the use of DOE for solving inverse problems, obtaining the solution, analysis of the design space and comparison of both problems under exact and noisy measurement data.

The paper is organized as follows. Section 2 presents the numerical model used for solving direct bioheat problems, together with the two numerical examples under investigation. Section 3 describes and discusses the steady-state and transient inverse bioheat problems, the evaluation of the objective function and the DOE approach. Section 4 discusses the results obtained for both inverse problems under exact and noisy measurement data, together with a detailed discussion of the inverse problem results. Finally, Section 5 presents the conclusions of the work with its most important results.

\section{Numerical model}

To investigate the inverse problem of estimating the size and material properties of skin tumour based on the skin surface temperature, we need to define the numerical model, as well as numerical examples upon which the inverse analysis will be done. The numerical model is based on the work of Bhowmik et al. [39,41], Çetingül and Herman [26] and Cheng and Herman [32] that treated the bioheat transfer problem in skin considering epidermis, papillary dermis, reticular dermis, fat and muscle tissue, where the tumour has been placed inside the papillary dermis. Therefore, the numerical model can describe heat transfer in skin containing a tumour more accurately than the simplified models considering only the lesion and uniform surrounding tissue.

Although different layers of tissue will be considered in the numerical model, there is still some room for improvement to describe thermal and pathological behaviour of the skin, malignant melanoma lesion and surrounding tissue together with some response mechanisms more realistically, especially for the cooling-rewarming process. We could include more realistic behaviour by adding the inflammation of tissue around the tumour blending the tumour boundary, temperature response of the skin (skin contraction under cooling), blood flow and water content in tissue which affects the thermal properties like tissue heat storage capacity, as well as pathological changes of the tumour in advanced stages. These mechanisms are known, however, for being very difficult to model because of the lack of accurate mathematical models due to difficult validation. Therefore, for this investigation, the numerical model will be based on Pennes bioheat equation with constant material properties, that will make the model simpler and also the analysis comparing different tumour sizes and the difference between the steady-state and transient inverse problems easier. In this section, we will describe the governing equation for bioheat transfer and the numerical examples used in solving inverse problems. 


\subsection{Bioheat transfer equation}

To describe heat transfer in biological tissue, the Pennes model [42] has been used due to its simplicity and acceptance by many researchers in this field. The governing equation can be written as

$$
\rho c_{p} \frac{\partial T}{\partial t}=\vec{\nabla} \cdot(\lambda \vec{\nabla} T)+\omega_{b} \rho_{b} c_{p, b}\left(T_{a}-T\right)+q_{m},
$$

where $T$ represents tissue temperature, $\rho, \lambda$ and $c_{p}$ the effective tissue density, thermal conductivity and specific heat, respectively, $\omega_{b}$ is blood perfusion rate, $\rho_{b}$ density of the blood, $c_{p, b}$ is specific heat of the blood, $T_{a}$ represents arterial blood temperature, $t$ time and $q_{m}$ metabolic heat source. The governing equation is a parabolic partial differential equation, where the term on the left side represents heat storage, while the terms on the right side represent heat diffusion and two heat sources due to blood perfusion and cell metabolism. Blood perfusion represents the volumetric blood flow rate through the capillary network and small arterioles per tissue volume and is therefore, non-directional. The assumption here is that the whole heat transfer between the arterial blood flow and surrounding tissue happens on the capillary level due to the large interface surface. As can be seen, the perfusion heat source term depends on the tissue temperature and therefore acts also like a heat sink, depending on the temperature difference between the tissue and the arterial blood. For this reason, the blood flow through the tissue can warm up or cool down the tissue. This term has also the highest effect on bioheat transfer because of its magnitude, while the magnitude of the heat source due to the cell metabolism is lower.

Carcinogenic tissue is well known to have a much greater blood perfusion due to oxygen and nutrition demand than normal tissue and therefore, the magnitude of perfusion heat generation is also larger, which is reflected in the higher tissue temperature. In arteriosclerosis or other arterial or capillary lesion where the blood perfusion is lowered, this can be manifested in lower temperature of the affected tissue. For this reason, we can connect tissue temperature with tissue physiology and improve the diagnostic procedure for lesions, which is the aim of this paper.

It is also known that blood perfusion is temperature dependent, because of the dilatation and contraction temperature reaction of the vessels. However, due to the lack of an exact temperature reaction model, as previously mentioned, the blood perfusion in this paper will be treated as constant. The arterial blood temperature will also be treated as constant and equal to the body core temperature, which is acceptable for the resting position of the person during the diagnostic procedure.

The numerical model in this paper will contain several different layers of tissue, considering constant material properties for each layer. Therefore, equation (1) has to be considered for each layer separately and connected together by compatibility and equilibrium conditions at the interface. With known boundary and initial conditions, a direct bioheat problem can be solved. The result from solving direct bioheat problems is the temperature distribution for the whole computational domain. For solving inverse problems, we will need only the response temperature at the skin surface. 


\subsection{Direct bioheat problem examples}

As stated, this paper covers the solution and analysis of two inverse problems to estimate the melanoma size and its physiological parameters. The first one is based on the steadystate measurement of the skin surface temperature, while the second uses transient skin surface temperature measurements during a cooling-rewarming process as described by Çetingül and Herman [26]. Therefore, we have to solve two different direct bioheat problems to obtain the skin surface temperature under steady-state and transient conditions. In the steady-state case the temperature is independent of time and for this reason the time derivative in equation (1) vanishes.

The analysis of both inverse problems will be done on two different melanoma sizes, Clark II and Clark IV. Therefore, we will be able to compare how the stage of melanoma affects the diagnostic procedure or the success of parameter estimation. Clark et al. [2] proposed a classification of melanoma into five levels, depending on their thickness or penetration depth. Clark II level tumour penetrates into the papillary dermis, while Clark IV penetrates into the reticular dermis. Regardless of the tumour thickness, its diameter can vary. However, the probability that the Clark IV melanoma has a larger diameter is higher $[41,43]$.

Both direct problems were treated as 2D, considering epidermis, tumour (melanoma), papillary dermis, reticular dermis, fat and muscle tissue. The computational domain for Clark II and Clark IV melanoma can be seen in figure 1, where the melanoma lesion is always placed in the center of the domain. The thickness of each layer and melanoma size are gathered in table 1 , together with the material properties. The thickness and diameter were chosen based on the literature review and the metabolic heat generation for the tumour has been rounded from $3680 \mathrm{~W} / \mathrm{m}^{3}$ to $3700 \mathrm{~W} / \mathrm{m}^{3}$. The material properties for each tissue vary and were not exactly determined, as stated in the work of Çetingül and Herman [43]. Çetingül and Herman [26,43] and other authors [32,41] upon which this model is based took an average value.

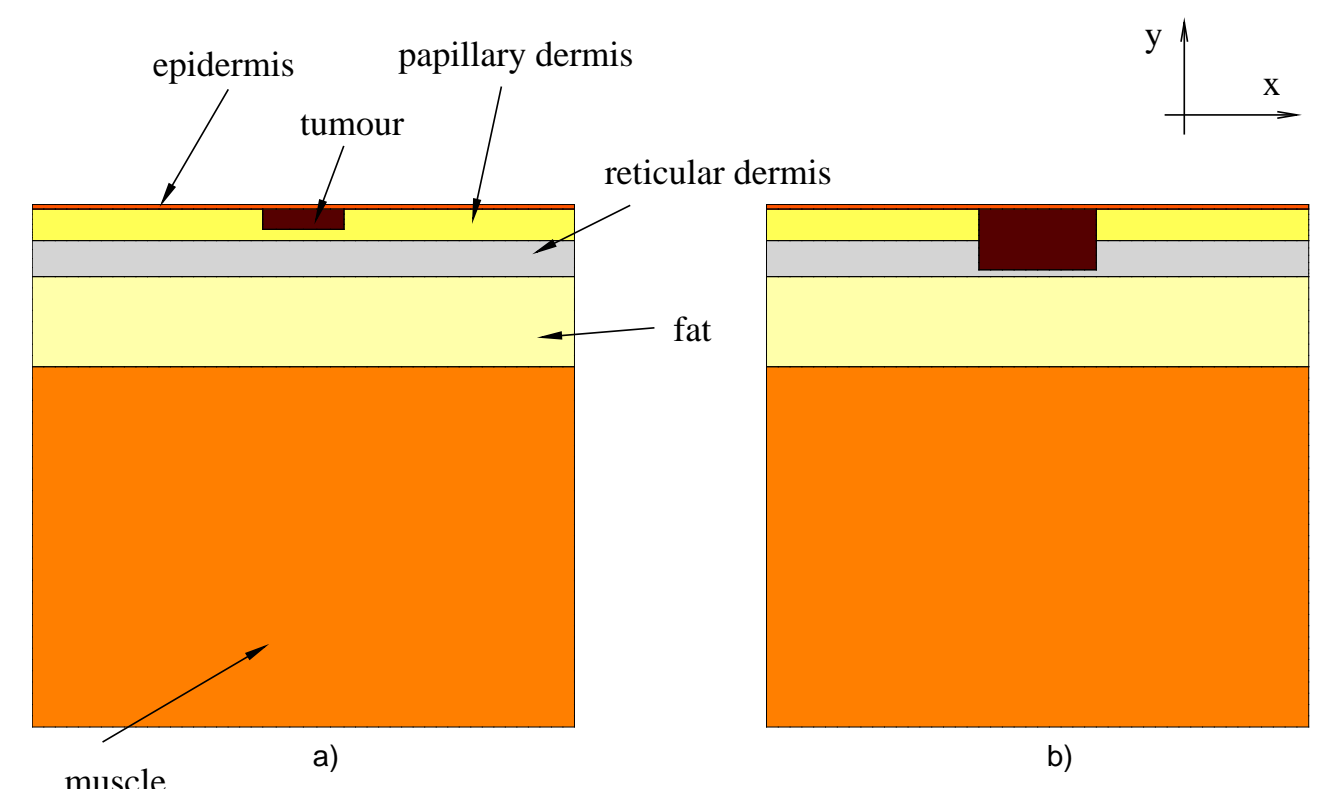

Figure 1: Multilayer computational domain: a) Clark II and b) Clark IV melanoma. 


\begin{tabular}{c|cc|ccccc} 
Material & $\Delta x[\mathrm{~mm}]$ & $\Delta y[\mathrm{~mm}]$ & $\rho\left[\mathrm{kg} / \mathrm{m}^{3}\right]$ & $c_{p}[\mathrm{~J} / \mathrm{kgk}]$ & $k[W / \mathrm{WK}]$ & $\omega_{b}[\mathrm{I} / \mathrm{s}]$ & $q_{m}\left[\mathrm{~W} / \mathrm{m}^{3}\right]$ \\
\hline Epidermis & 12 & 0.1 & 1200 & 3589 & 0.235 & 0.0 & 0.0 \\
Papillary dermis & 12 & 0.7 & 1200 & 3300 & 0.445 & 0.0002 & 368.1 \\
Reticular dermis & 12 & 0.8 & 1200 & 3300 & 0.445 & 0.0013 & 368.1 \\
Fat & 12 & 2.0 & 1000 & 2674 & 0.185 & 0.0001 & 368.3 \\
Muscle & 12 & 8.0 & 1085 & 3800 & 0.510 & 0.0027 & 684.2 \\
\hline Tumour - Cl. II & 1.8 & 0.45 & 1030 & 3852 & 0.558 & 0.0063 & 3700 \\
Tumour - Cl. IV & 2.6 & 1.35 & 1030 & 3852 & 0.558 & 0.0063 & 3700 \\
\hline Blood & - & - & 1060 & 3770 & - & - & -
\end{tabular}

Table 1: Material properties with tissue dimensions [26,32, 41].

The arterial blood temperature is usually treated as equal to the body core temperature, between $36.5-37.5^{\circ} \mathrm{C}$. Therefore, in this paper we are assuming a constant arterial blood temperature of $T_{a}=37.0^{\circ} \mathrm{C}$ for the steady-state, as well as the transient cooling-rewarming problem.

To solve the direct bioheat problem we also have to prescribe boundary conditions. For both problems, we prescribe Neumann boundary conditions on the left and right sides of the computational domain; $q=0 \mathrm{~W} / \mathrm{m}^{2}$, while on the bottom side the Dirichlet condition equal to the body core temperature $T=T_{a}=37.0^{\circ} \mathrm{C}$. For the steady-state problem a constant Robin boundary condition has been applied at the skin surface, taking into account natural convection, evaporation and thermal radiation; $\alpha=10 \mathrm{~W} / \mathrm{m}^{2} \mathrm{~K}, T_{\infty}=$ $22.4^{\circ} \mathrm{C}$. For the simulation of the cooling-rewarming process, the boundary condition changes through time. During the first minute of the process the Dirichlet boundary condition is prescribed; $T=13^{\circ} \mathrm{C}$, while for the rest of the simulation the already given Robin boundary condition is assigned. During the first period we are simulating the cooling process of the skin tissue, while after the first minute the skin is exposed to the environment and the rewarming process occurs. The rewarming period takes a long time to reach the steady-state condition, however, we simulated only ten minutes of it to capture the most rapid change in the skin temperature. After ten minutes, the temperature change is small. Therefore, the total simulation time for transient problems has been 11min. The boundary conditions have been taken from the work of Çetingül and Herman [26].

For a steady-state problem we do not need to prescribed any initial conditions, while for the cooling-rewarming process the solution from a steady-state simulation has been given as the initial condition.

The steady-state and transient bioheat problems for Clark II and Clark IV melanoma has been solved to obtain the temperature response at the skin surface, which has been treated as a measurement data for the inverse problems. To mimic real measurement data we add a white noise to the temperature response as

$$
T_{m, k, p}=T_{s, k, p}+\Delta T_{m} \cdot r
$$

where $r$ represents a random number; $r \in[-1,1], \Delta T_{m}$ is temperature deviation or noise, index $s$ stands for simulated temperature, $m$ for measurement temperature and $k$ and $p$ for time and location of the measurement, respectively. Luna et al. considered a temperature deviation of $60 \mathrm{mK}$ [35] and $50 \mathrm{mK}$ [36], while Bhowmik and Repaka considered three 
different levels; $10 \mathrm{mK}, 50 \mathrm{mK}$ and $100 \mathrm{mK}$ [39]. Hatwar and Herman [30] used a random noise of $10 \mathrm{mK}$, which is very low and difficult to obtain in practice. Çetingül and Herman stated that their equipment has a resolution of $25 \mathrm{mK}$ [43], which is possible to obtain using laboratory equipment. Modern IR cameras can obtain the NETD (Noise Equivalent Temperature Difference) value of less than $30 \mathrm{mK}$. Thus, we investigate both inverse problems under three different noise levels; $0 \mathrm{mK}, 25 \mathrm{mK}$ and $50 \mathrm{mK}$. The first one represents exact measurement data, while the last two represent low and high level of noise. In the last two cases, the measurement data do not follow the numerical model anymore.

\section{$2.3 \quad$ Solver}

For solving the direct bioheat problems we used a numerical solver based on the subdomain Boundary Element Method (BEM) and mixed elements. Quadratic interpolation elements were used for the temperature, while constant interpolation elements were used for the normal derivative. The elliptic fundamental solution is used for the steady-state problem, while for the transient one a parabolic fundamental solution is used to speed-up the computational time. The speed-up is also made by implementing the sub-domain approach, comparing to the classical BEM approach. The computational time, especially for transient problems, has been drastically reduced as described in our previous paper [40]. The developed solver is fast with high accuracy, which is needed for solving inverse problems. As the aim of this paper is to analyze two different inverse bioheat problems, a detailed description of the solver can be find in [40] and is, therefore, omitted here.

\section{Inverse problems}

Çetingül and Herman [26] proposed the cooling-rewarming technique for pigmented lesion to identify if they are malignant by recording the temperature response with an IR camera. The mechanism behind this process is that a malignant pigmented lesion will warm up much quicker than the surrounding healthy tissue, and we can observe the temperature difference with an IR camera more easily. The steady-state temperature difference can be too small to identify malignant melanomas, especially at an early stage.

The idea behind this paper is to identify several parameters of malignant melanoma lesions based on the skin surface temperature response, and to compare the success of parameter estimation between the steady-state temperature measurement (static thermography) and transient cooling-rewarming measurement (dynamic thermography). Therefore, we solve two different inverse problems. The parameters that we will try to identify are diameter, thickness, blood perfusion rate and metabolic heat generation of the tumour. The inverse problems of parameter estimation have been solved numerically, using an optimization approach by defining the objective function, which represents the difference between the temperature measurement and the temperature response from the direct numerical model. Most authors in this field used stochastic optimization algorithms such as Genetic Algorithms (GA) and Simulated Annealing (SA) [33-36,39] to solve their optimization problems, however in this paper the full factorial DOE is used not only to solve the inverse problems but also to analyse the design space, obtain the parameter sensitivity and model response, as well as to compare the static and dynamic 
thermography performance.

The solution of the optimization problem is represented by the value of parameters when the objective function is at a minimum. Some optimization problems have more than one global minimum, which is undesirable for inverse problems as this means that the solution is non-unique. For both inverse problems treated in this paper, we could not find the mathematical background to prove the solution uniqueness, which boundary conditions are needed or some other constraints to render a unique solution.

Therefore, this paper analyzes how the type of temperature measurement and stage of malignant melanoma affect the estimation of important tumour parameters. This is done for exact as well as noisy measurement data that has been numerically obtained. The reason to investigate the inverse problem on simulated measurement data is because the exact value of parameters that should be obtained are known. It should also be stated here that under noisy measurement data, there is no unique solution of the inverse problem because the measurement data do not follow the numerical model anymore. In the case of one global minimum or unique solution under exact measurement data, it is hoped that the deviation of the estimated parameters is not too large under noisy measurement data.

\subsection{Objective function}

As stated, the inverse bioheat problems are transformed to optimization problems by defining the objective function, which represents the difference between the measurement and response obtained from solving the direct problem for given values of parameters. For both inverse problems in this paper, the objective function can be written as

$$
F\left(d, h, \omega_{b}, q_{m}\right)=\frac{1}{2} \sum_{k=1}^{n_{t}} \sum_{p=1}^{n_{p}}\left(T_{s, k, p}-T_{m, k, p}\right)^{2},
$$

where $F(\cdot)$ represents the value of the objective function, $d$ the diameter of the tumour, $h$ its thickness, $\omega_{b}$ the blood perfusion of the tumour, $q_{m}$ the metabolic heat generation of the tumour, $T_{s}$ stands for the simulated skin surface temperature, $T_{m}$ the measurement, index $k$ and $p$ the time position and location of the temperature measurement or model response, respectively, and $n_{t}$ and $n_{p}$ the number of time and locational measurements or observations, respectively.

We compare the skin temperature response with measurements of $n_{p}=30$ equidistant points along the skin surface. For the steady-state case the number of time measurements is one, therefore $n_{t}=1$, while for the cooling-rewarming case time observations were taken each second for the rewarming period, which means $n_{t}=600$ time observations. Therefore, the number of data comparisons for the steady-state case is 30 , while for the transient case it is 18.000 , which will lead to different values of the objective function. The steady-state skin temperature measurement that has been taken for inverse problem analysis can be seen in figure 2 for both melanomas, while figures 3 and 4 show the skin surface temperature response to the cooling-rewarming test. As can be observed from steady-state skin temperature, the temperature difference between the center of tumour and health skin is smaller for Clark II compared to the Clark IV size, and also that the temperature difference is in the range of $0.03-0.07 \mathrm{~K}$, which is hard to detect or to measure. The results for the cooling-rewarming test show that the temperature difference 
is much greater during the beginning of the rewarming stage, even for the Clark II size $(0.25 K)$. Here, we can observe the advantage of the cooling-rewarming test, because it is possible to identify small malignant melanoma in its early stage using IR cameras fairly easily. Figures 2, 3 and 4 represent the exact as well as noisy temperature measurements for Clark II and Clark IV melanoma. It can be observed that the measurement noise under steady-state condition is almost comparable to the temperature difference between tumour and healthy tissue, while for the transient measurement it does not affect the temperature distribution or shape profile.
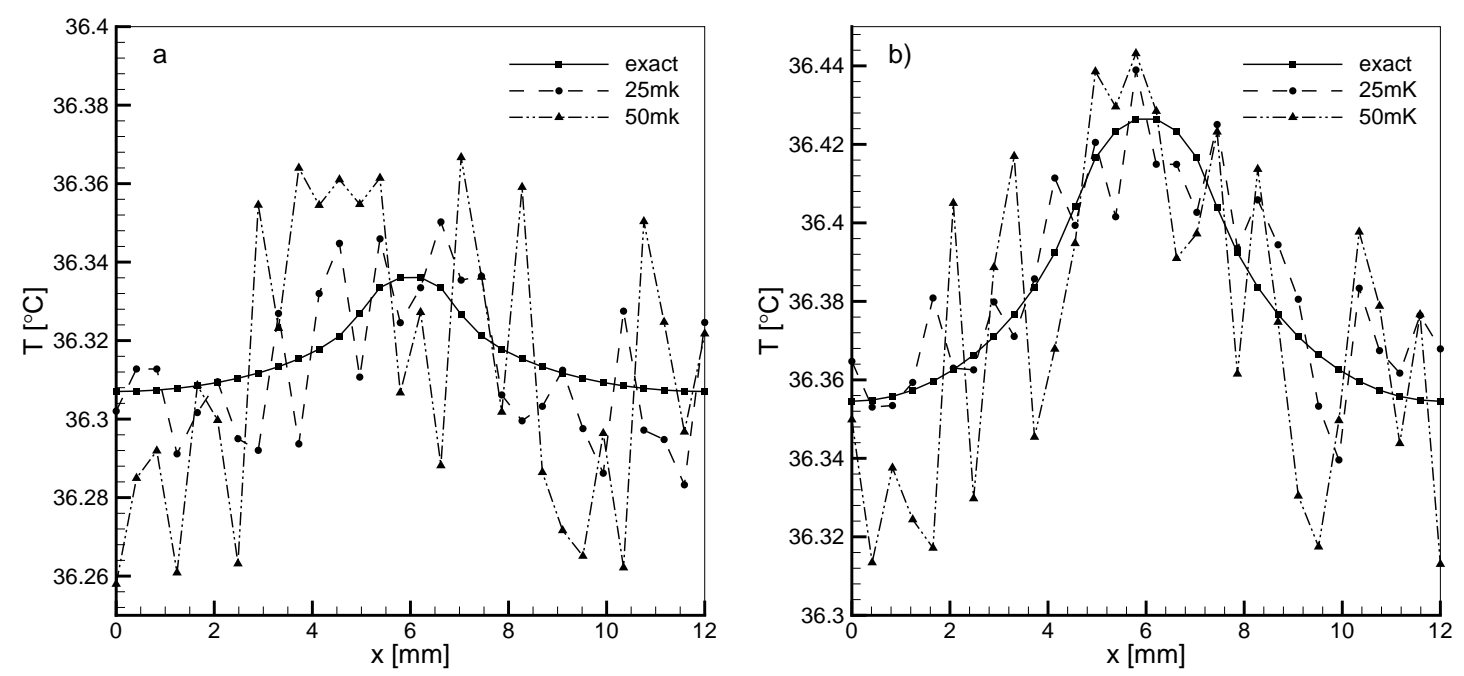

Figure 2: Exact and noisy steady-state skin temperature response: a) Clark II and b) Clark IV lesion size.

As already stated, the objective function has been evaluated numerically using our own accurate and fast solver [40]. If the numerical accuracy for solving the direct problem is low, this can lead to a poorly defined objective function which is no longer smooth and can lead to a local minimum. In this case, it is very hard to use gradient based optimization algorithms resulting in the need for accurate numerical solvers for obtaining a smooth objective function.

\subsection{Design of Experiment}

Design of Experiment (DOE) is a mathematical method for systematically planning and conducting numerical or measurement experiments by changing the input variables to obtain the model response. The main objectives are to get the input variable sensitivity and to obtain the model response [44-46]. This is the main reason why the DOE has been used in this paper.

There are many DOE techniques to choose appropriate designs for model analysis like Randomized Complete Block Design (RCBD), Fractional Factorial Design (FFD), Box-Behnken design, Taguchi design, etc. The DOE is used to obtain an initial design of experiment to obtain the model response upon which the meta-model or surrogate model (SM) is built for faster optimization. Response Surface Models (RSM) are the most 

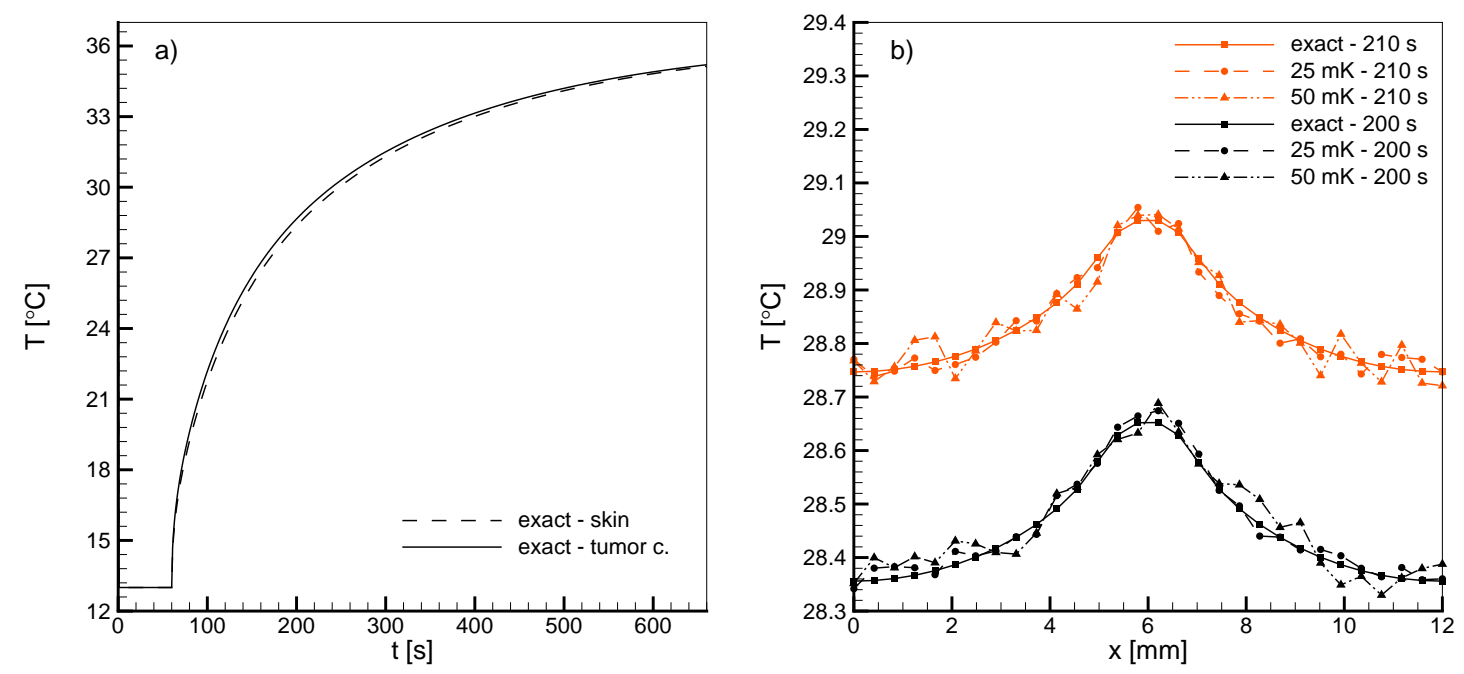

Figure 3: Transient skin temperature response to cooling-rewarming test for Clark II melanoma size: a) exact temperature time change for healthy skin and center of the tumour and b) exact and noisy surface temperature representation for different times.
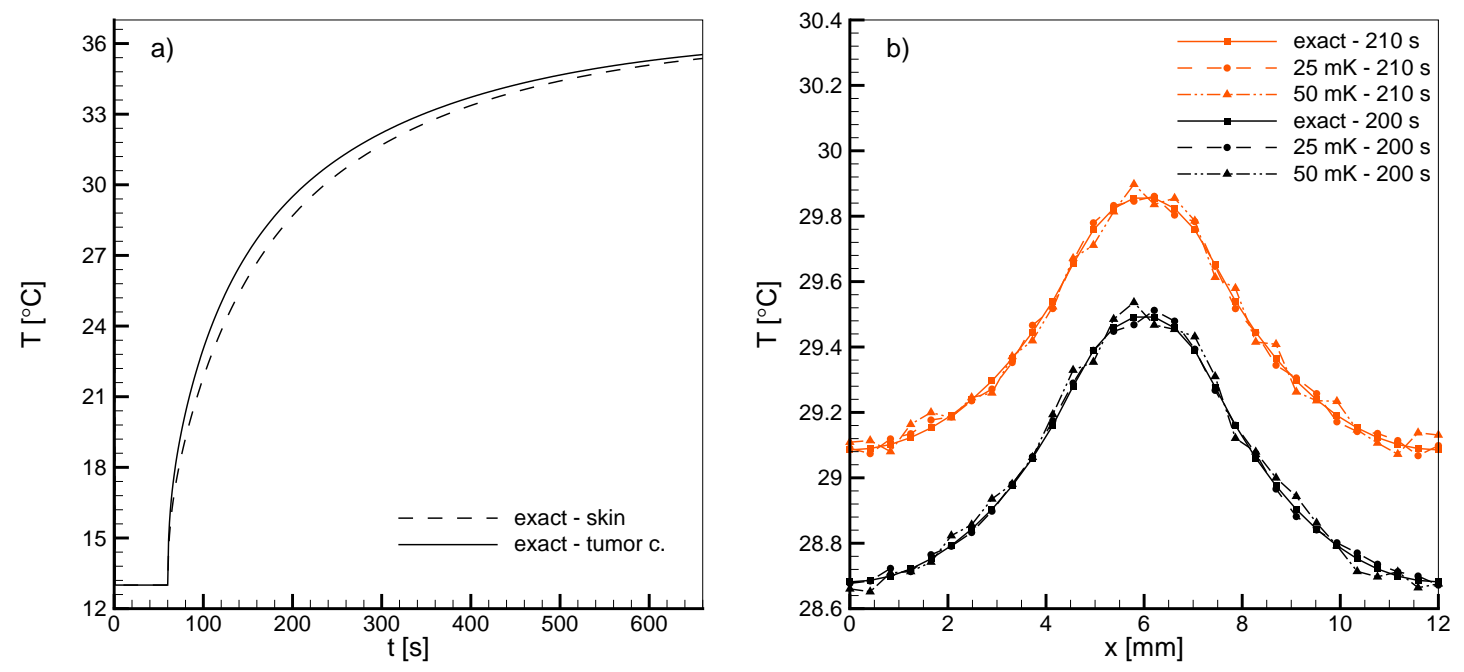

Figure 4: Transient skin temperature response to cooling-rewarming test for Clark IV melanoma size: a) exact temperature time change for healthy skin and center of the tumour and b) exact and noisy surface temperature representation for different times.

popular SM in general. Based on the initial model response (initial design of experiment) or response surface the position of the minimum is obtained from current population or estimated from the response surface. Based on the initial DOE, new designs can be added for further evaluation of the model response and to find better designs with lower value of the objective function. The number of generations can be one or greater than one depending on the conditions. The DOE is mostly used in engineering problems with 
complex computational models that have high computational cost [45-48]. For better understanding of the DOE method, figure 5 shows the arbitrary model response using two input variables $\left(x_{1}\right.$ and $\left.x_{2}\right)$ for two different initial DOE, the full factorial and the RCBD. As can be seen, the number of designs for the full factorial DOE is much higher compared to the RCBD, and is therefore computationally more expensive. However, on the other hand, the accuracy of the response model is better for the whole design space. Usually, to save computational time, the model is only evaluated at certain points and then SM models are used to evaluate the response model at non-investigated design space to find the minimum of the problem. Figure 5 also shows the obtained response surface for RCBD and evaluated position of the minimum. Comparing the response surface with the actual model response (full factorial DOE), we can observe that the response surface can have a good agreement in some regions but cannot find the real position of the minimum. Therefore, SM models are appropriate to use when the model response is smooth and monotonic, otherwise the error can be high and a higher number of evaluated designs is needed. Based on the calculated model response, the optimum is then selected based on the minimum value of objective function for the current evaluated designs.

a)

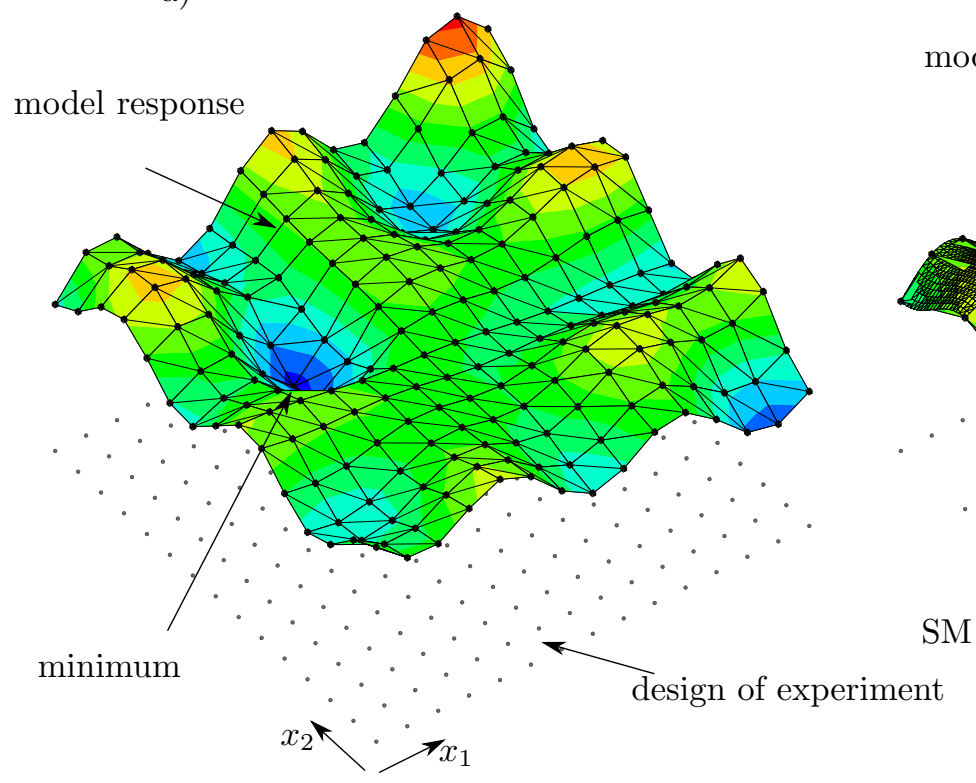

b)

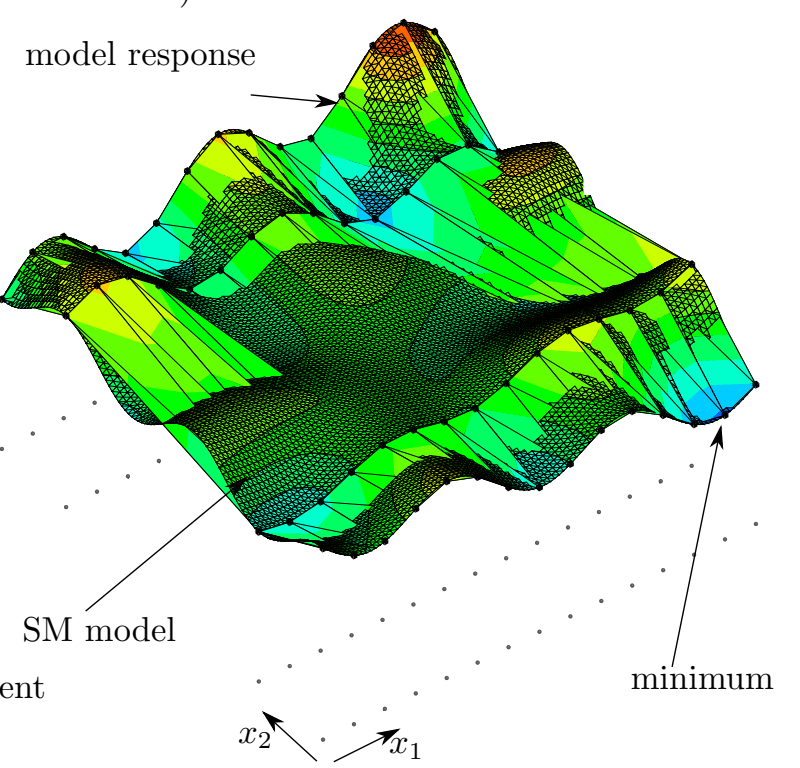

Figure 5: DOE and response for arbitrary model using different techniques: a) full factorial DOE and b) RCBD DOE with SM surface.

Because a high accuracy of the model response is needed the general full factorial DOE without SM is used in this paper. The SM is an approximation of the numerical model (figure 5) and can lead to the wrong solution or conclusion, and thus should be avoided for solving inverse problems. The full factorial DOE using a high number of levels for each parameter has been used to investigate the whole design space and to make the sensitivity analysis of both inverse problems. The full factorial DOE is the simplest algorithm for solving optimization problems, however it is rarely used because of the high computational costs, especially when solving complex systems involving partial differential equations, and also because data processing can be time consuming. The number 
of levels or subdivisions for each parameter has to be high enough to get appropriate objective function sensitivities, which leads to a high number of model evaluations and high computational costs.

The range of searched variables were taken as follows:

$$
\begin{aligned}
\omega_{b}\left[s^{-1}\right] & \in[0.001,0.01], \\
q_{m}\left[W / m^{3}\right] & \in[1500,5100], \\
d[m m] & \in[0.5,5.0], \\
h[m m] & \in[0.1,1.5],
\end{aligned}
$$

where the number of levels or subdivisions of individual parameters has been chosen based on the importance of the resolution. The blood perfusion rate space has been subdivided by the step $\Delta \omega_{b}=0.0001 \mathrm{~s}^{-1}$, the metabolic heat generation by the step size $\Delta q_{m}=200 \mathrm{~W} / \mathrm{m}^{3}$, the tumour diameter by the step $\Delta d=0.1 \mathrm{~mm}$ and the tumour thickness by $\Delta h=0.05 \mathrm{~mm}$. This means that the number of levels for the blood perfusion rate is 91 , for metabolic heat generation 19, for tumour diameter 46 and for thickness 29. This leads to 2,306, 486 numerical model evaluations for steady-state and the same amount for transient inverse problems. The full factorial design is rarely used for this high number of levels or parameters. The number of model evaluations in engineering problems using FFD DOE is usually between 30 and 150 [45-48], which is much less than in this study.

Computational time for one design evaluation depends on the numerical model, adopted solver and CPU, which in our case was on average 116.69s for transient and 8.49s for a steady-state problem. The CPU used was Intel Xeon E5-2670 at our HPC-Core cluster. This means that the total computational cost for the full factorial DOE for the transient inverse problem would be $74,762.18 \mathrm{~h}$ and for the steady-state inverse problem $5,439.46 \mathrm{~h}$ if the problems were solved on a single computer. To reduce the total computational time, design evaluations can be solved independently using parallel computing. We used 100 parallel cores to reduce the total time to 31.15 days for the transient case and 2.26 days for the steady-state one. As can be seen from this discussion, the total computational time or cost is not small and, for this reason, full factorial DOE is not an appropriate method when fast optimization is required. However, the total time can be reduced by the number of available parallel computers or cores. The main reason for using this approach is to investigate the design space under exact and noisy measurement data, to assist the selection of the appropriate deterministic or stochastic optimization technique for future calculations.

\section{Results and discussion}

The evaluation of the model response using full factorial DOE has been done for all four parameters at predetermined points or designs, to obtain the response surface for each inverse problem. Because of the four parameters, the searched design space is $4 \mathrm{D}$ and can therefore be hard to represent. For this reason, we show the objective function value (response surface) using 2D slice plots of the 4D space by fixing two parameters, while the solution to the inverse problems using DOE is gathered in tables. The solutions to the inverse problems are presented by their three best results, which gave the lowest value of 
the objective function for the searched space. We also investigate both inverse problems for different scenarios, if it would be easier to estimate the parameters if some of them are known, especially for steady-state cases under measurement noise. We assume this will explain some of the problems regarding to the steady-state measurement approach and how many parameters can be evaluated using it.

The steady-state inverse problem results are initially presented and discussed, followed by the transient problem results and findings. For better representation of the response surface and comparison between different problems, the value of the objective function has been transformed (scaled) by using a scaled logarithmic value in the form

$$
F_{T}=\frac{\log (F)-\log (\min [F])}{\log (\max [F])-\log (\min [F])}
$$

where $F_{T}$ represents the transformed value of the objective function, $F$ the value of the objective function for each DOE design, while arguments $\min [\cdot]$ and $\max [\cdot]$ represent the minimum and maximum value, respectively. The minimum and maximum values of the objective function have been picked from the whole design space for each inverse problem. The 2D plots also contain a diamond shaped dot representing the position of the exact solution (exact parameters) for better visual inspection.

\subsection{Steady-state results}

The metabolic heat generation is investigated first, using steady-state measurement data. Figure 6 represents the response surface for exact measurement data, correlating the metabolic heat generation and blood perfusion rate for a Clark II tumour at different thicknesses and tumour diameter $1.8 \mathrm{~mm}$. The dot in the figure represents the exact solution for a metabolic heat generation of $3700 \mathrm{~W} / \mathrm{m}^{3}$ and a blood perfusion rate of $0.0063 \mathrm{~s}^{-1}$. As can be observed from the figure, the isolines are very straight in the direction of metabolic heat generation, which indicates low sensitivity. The isolines are also leaning towards a reduction in blood perfusion rate, which indicates that higher metabolic heat generation estimation will lead to lower blood perfusion rate detection, which is the response of the inverse problem. When slicing the 4D design space using the exact value of tumour thickness $0.45 \mathrm{~mm}$, we can observe that we get a band of low objective functions, which coincides with the exact solution. When exploring the space with a different thickness from the exact one, we can observe a similar behaviour, however with higher values of the objective function at the position of the exact solution. The analysis of the objective function showed the same behaviour when looking at the correlation between the metabolic heat generation and thickness or diameter of the tumour, as well as for the Clark IV tumour, and is therefore omitted here. We can conclude from this initial study that the sensitivity of the metabolic heat generation is small and it will be difficult to estimate its exact value using gradient based optimization techniques, as has been stated by Çetingül and Herman [26] and Bhowmik and Repaka [39].

Therefore, for our further analyses on blood perfusion, tumour thickness and diameter we fixed the metabolic heat generation, reducing the $4 \mathrm{D}$ solution space to $3 \mathrm{D}$. This simulates the case when only three parameters are unknown and the metabolic heat generation is predetermined or already evaluated. Figures 7 and 8 show the response surface for Clark II and Clark IV tumours using exact measurement data, correlating the blood perfusion and tumour thickness at several different diameters. As can be 
a)
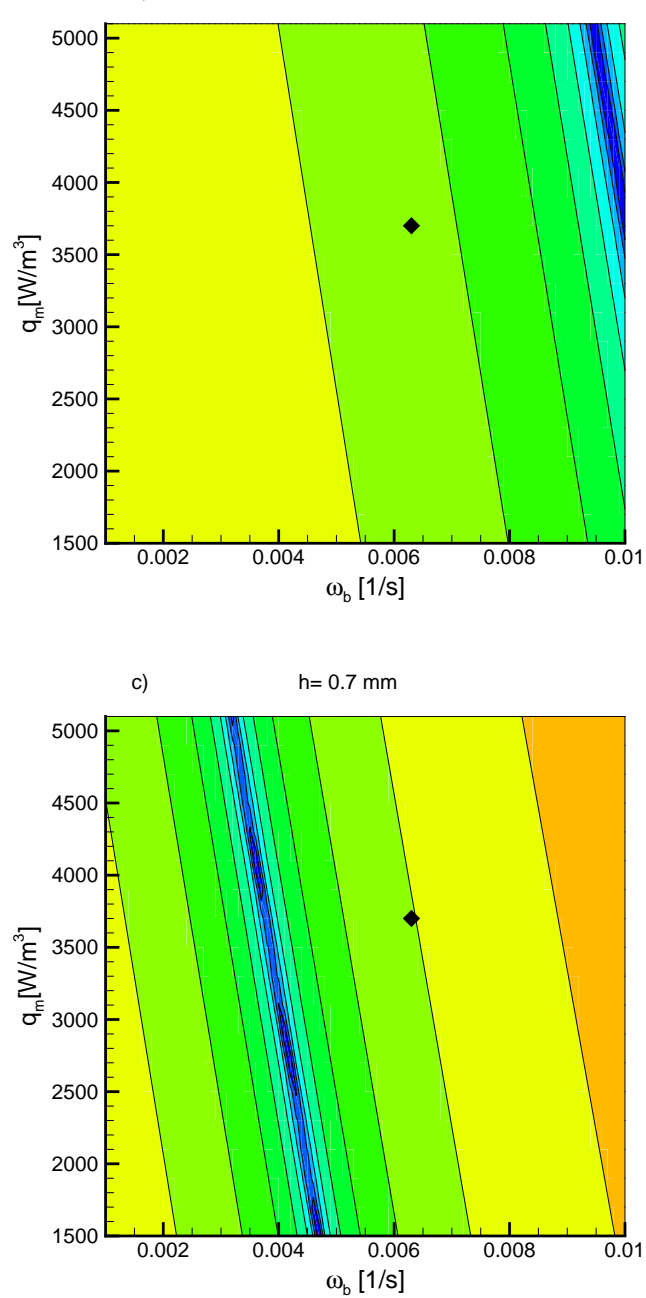

b)
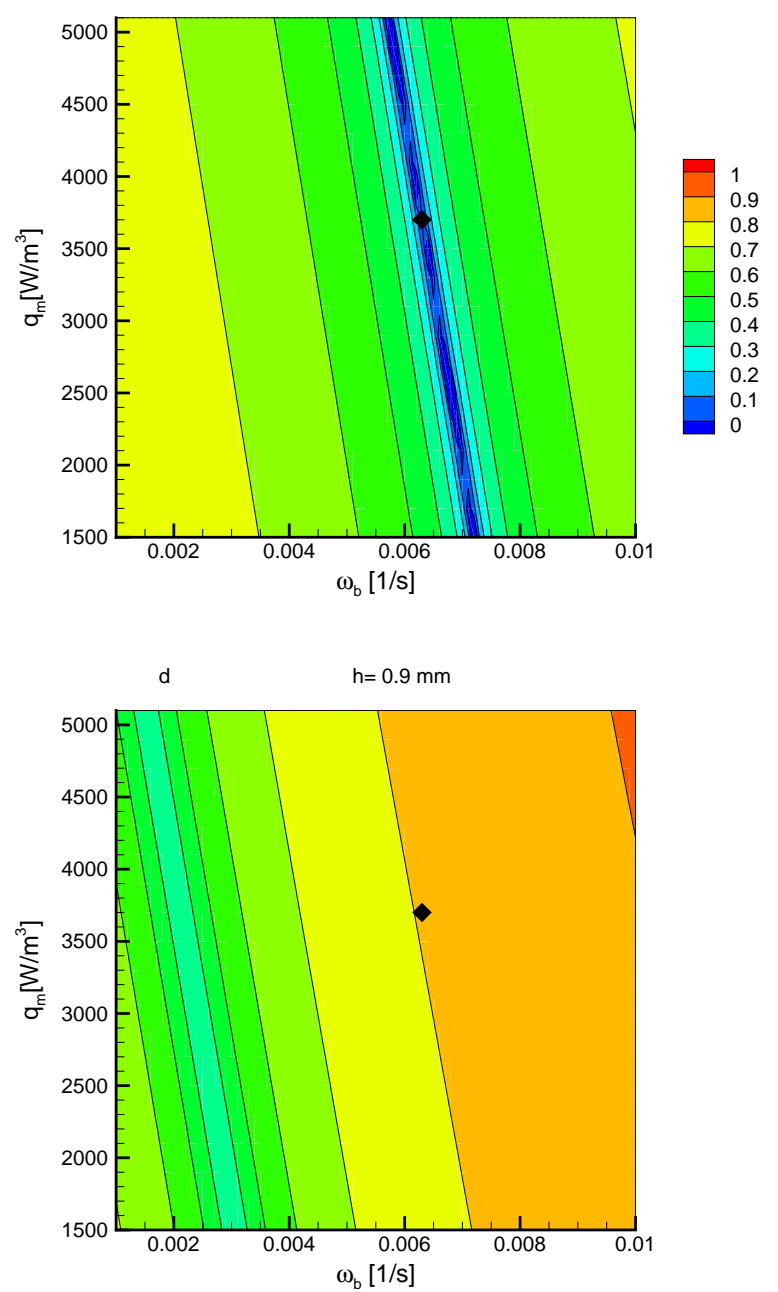

Figure 6: Response surface of objective function for exact measurement data comparing metabolic heat generation and blood perfusion rate for Clark II tumour at exact diameter $1.8 \mathrm{~mm}$ and different tumour thicknesses: a) $h=0.3 \mathrm{~mm}$, b) $h=0.45 \mathrm{~mm}$, c) $h=0.7 \mathrm{~mm}$ and d) $h=0.9 \mathrm{~mm}$.

seen from the plots for the exact diameter $1.8 \mathrm{~mm}$ for Clark II and $2.6 \mathrm{~mm}$ for Clark IV, the correlation is strong, because there is a band of low objective function values. It might seem from figures 7 and 8 that there are several local minima, however this happens because of the linear interpolation between two adjoint DOE designs, otherwise the objective function is smooth. To obtain a smoother plot we should increase the level of each parameter, leading to an even greater number of model evaluations. The only difference that can be observed between Clark II and Clark IV tumours is the value of the objective function gradient near the exact solution, which is greater for Clark IV than from Clark II, as can be seen in figures 7 and 8. A similar behaviour of the objective function is found between the blood perfusion and tumour diameter for constant tumour thickness, and is thus omitted here. As can be concluded from the analysis made on the response surface the correlation between the tumour diameter, thickness and blood 
perfusion is strong, while the metabolic heat generation is weakly correlated to the steadystate temperature response.
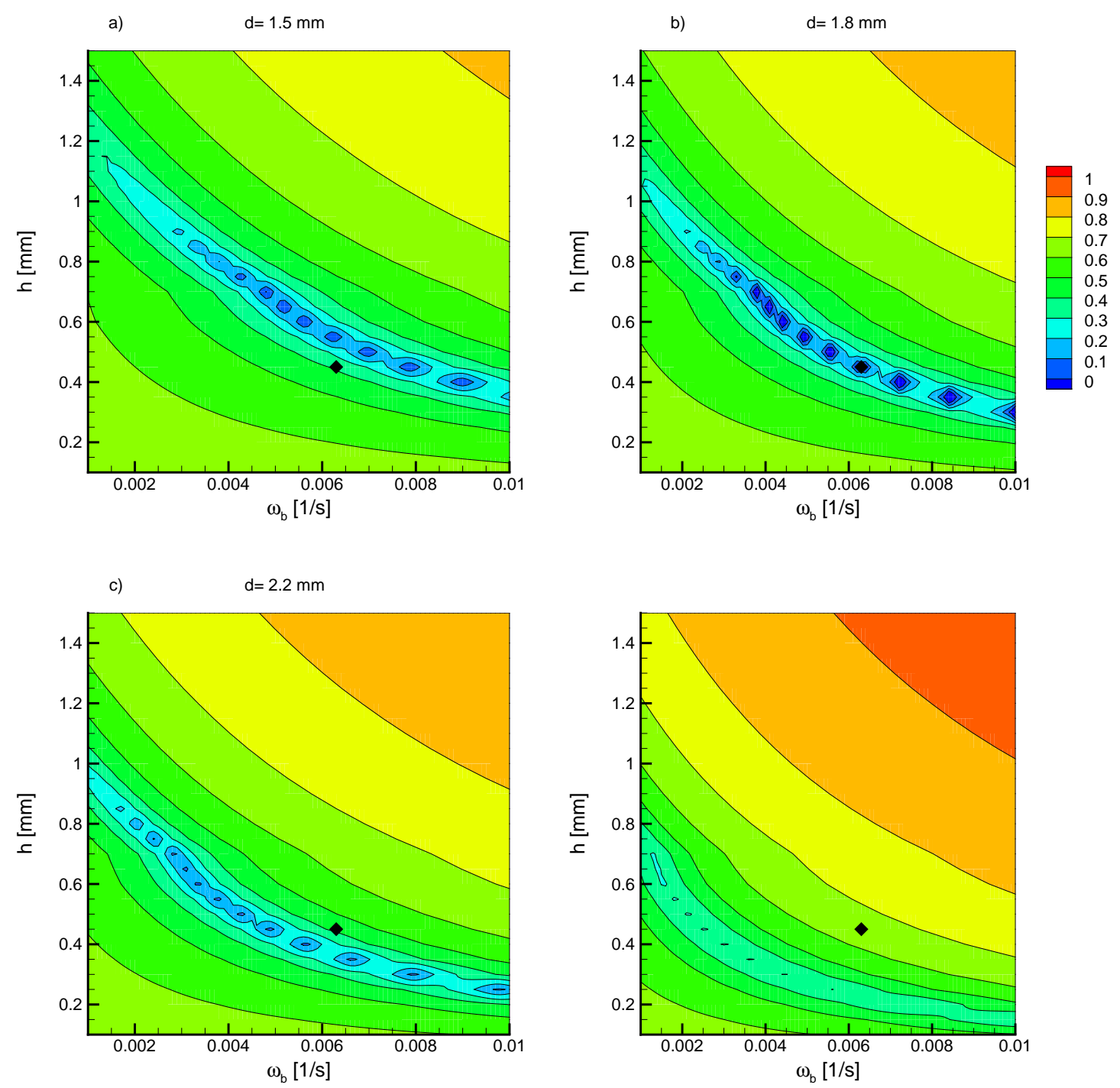

Figure 7: Response surface of objective function for exact measurement data comparing tumour thickness and blood perfusion rate for Clark II tumour at metabolic heat generation $3700 \mathrm{~W} / \mathrm{m}^{3}$ and different diameters: a) $d=1.5 \mathrm{~mm}$, b) $d=1.8 \mathrm{~mm}$, c) $d=2.2 \mathrm{~mm}$ and d) $d=3.5 \mathrm{~mm}$.

For this reason, table 2 shows the obtained results for the steady-state inverse problem using exact measurement data under known or estimated value of metabolic heat generation. Table 2 shows the three best solutions obtained by the DOE with corresponding values of the objective function. For exact data, the most obvious result is the retrieval of the exact solution with objective function zero. However, we can observe that the next best solutions having values of the objective function close to zero are far away from the exact solution for Clark II size, while they are much closer for Clark IV. This indicates that when using real steady-state measurement data it would be hard to estimate all 

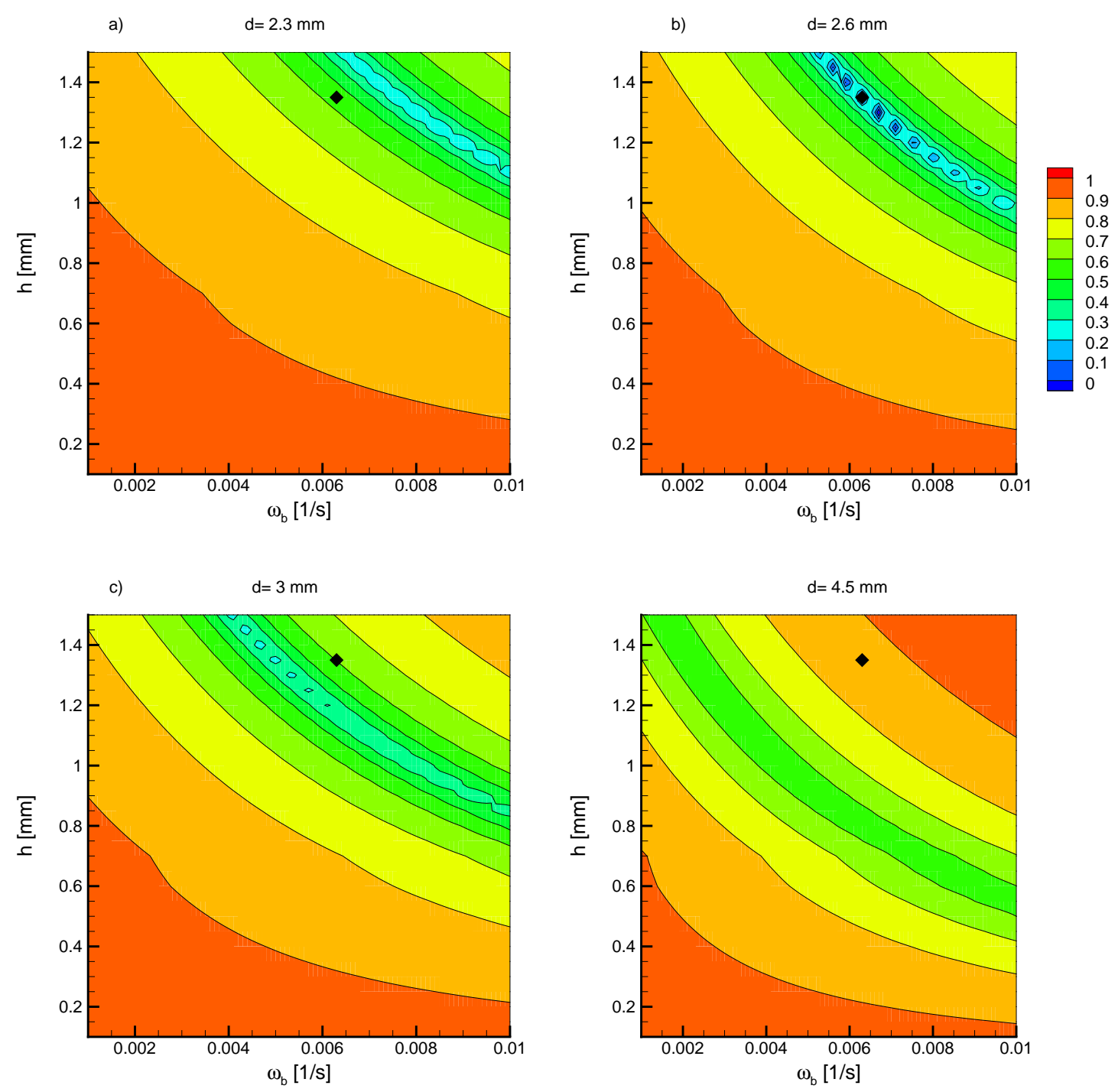

Figure 8: Response surface of objective function for exact measurement data comparing tumour thickness and blood perfusion rate for Clark IV tumour at metabolic heat generation $3700 \mathrm{~W} / \mathrm{m}^{3}$ and different diameters: a) $d=2.3 \mathrm{~mm}$, b) $d=2.6 \mathrm{~mm}$, c) $d=3.0 \mathrm{~mm}$ and d) $d=4.5 \mathrm{~mm}$.

three parameters simultaneously due to the measurement noise. In the case when we do not know the exact value of the metabolic heat generation, as shown in table 3 for $q_{m}=4500 \mathrm{~W} / \mathrm{m}^{3}$, the deviation between the estimated solution and the exact solution is inside a reasonable range. As can be seen, the solution oscillates around the exact solution.

Under a low level of measurement noise the objective function minimum is shifted, as can be seen from figures 9 and 10 for Clark II and Clark IV, respectively. We can observe that the region is further away from the exact solution for Clark II than for Clark IV. The deviation from the exact solution is even larger for the $50 \mathrm{mK}$ noise. Tables 4 and 5 show the obtained inverse solutions using measurement data with $25 \mathrm{mK}$ and $50 \mathrm{mK}$ 


\begin{tabular}{c|cccc|cccc} 
& \multicolumn{5}{|c|}{ Clark II } & \multicolumn{4}{c}{ Clark IV } \\
& $\omega_{b}\left[\mathrm{~s}^{-1}\right]$ & $h[\mathrm{~mm}]$ & $d[\mathrm{~mm}]$ & $F[-]$ & $\omega_{b}\left[\mathrm{~s}^{-1}\right]$ & $h[\mathrm{~mm}]$ & $d[\mathrm{~mm}]$ & $F[-]$ \\
\hline Exact & 0.0063 & 0.45 & 1.8 & - & 0.0063 & 1.35 & 2.6 & - \\
\hline Steady-state & 0.0063 & 0.45 & 1.8 & 0.0 & 0.0063 & 1.35 & 2.6 & 0.0 \\
& 0.0100 & 0.30 & 1.8 & $0.1107 \mathrm{E}-6$ & 0.0067 & 1.30 & 2.6 & $0.7262 \mathrm{E}-6$ \\
& 0.0084 & 0.35 & 1.8 & $0.1222 \mathrm{E}-6$ & 0.0059 & 1.40 & 2.6 & $0.2112 \mathrm{E}-5$ \\
\hline Transient & 0.0063 & 0.45 & 1.8 & 0.0 & 0.0063 & 1.35 & 2.6 & 0.0 \\
& 0.0057 & 0.50 & 1.8 & $0.5093 \mathrm{E}-2$ & 0.0065 & 1.35 & 2.5 & $0.8958 \mathrm{E}-1$ \\
& 0.0067 & 0.40 & 1.9 & $0.8232 \mathrm{E}-2$ & 0.0061 & 1.35 & 2.7 & 0.1082 \\
\hline
\end{tabular}

Table 2: Solution of the steady-state and transient inverse problem using exact measurement data and estimated metabolic heat generation $3700 \mathrm{~W} / \mathrm{m}^{3}$.

\begin{tabular}{c|cccc|cccc} 
& \multicolumn{5}{|c|}{ Clark II } & \multicolumn{4}{c}{ Clark IV } \\
& $\omega_{b}\left[\mathrm{~s}^{-1}\right]$ & $h[\mathrm{~mm}]$ & $d[\mathrm{~mm}]$ & $F[-]$ & $\omega_{b}\left[\mathrm{~s}^{-1}\right]$ & $h[\mathrm{~mm}]$ & $d[\mathrm{~mm}]$ & $F[-]$ \\
\hline Exact & 0.0063 & 0.45 & 1.8 & - & 0.0063 & 1.35 & 2.6 & - \\
\hline Steady-state & 0.0069 & 0.40 & 1.8 & $0.4688 \mathrm{E}-8$ & 0.0055 & 1.40 & 2.6 & $0.5463 \mathrm{E}-6$ \\
& 0.0046 & 0.55 & 1.8 & $0.1889 \mathrm{E}-7$ & 0.0055 & 1.35 & 2.7 & $0.3043 \mathrm{E}-5$ \\
& 0.0041 & 0.60 & 1.8 & $0.2589 \mathrm{E}-7$ & 0.0071 & 1.25 & 2.5 & $0.3707 \mathrm{E}-5$ \\
\hline Transient & 0.0070 & 0.40 & 1.8 & $0.17342 \mathrm{E}-2$ & 0.0063 & 1.35 & 2.6 & $0.8604 \mathrm{E}-1$ \\
& 0.0063 & 0.45 & 1.8 & $0.75564 \mathrm{E}-2$ & 0.0065 & 1.35 & 2.5 & 0.13867 \\
& 0.0066 & 0.45 & 1.7 & $0.96874 \mathrm{E}-2$ & 0.0061 & 1.40 & 2.6 & 0.17127 \\
\hline
\end{tabular}

Table 3: Solution of the steady-state and transient inverse problem using exact measurement data and estimated metabolic heat generation $4500 \mathrm{~W} / \mathrm{m}^{3}$.

noise, respectively, for known value of metabolic heat generation. As can be seen, under noisy steady-state measurement data the estimation of parameters is only possible for Clark IV and low level noise.

\begin{tabular}{c|cccc|cccc} 
& \multicolumn{5}{|c|}{ Clark II } & \multicolumn{4}{c}{ Clark IV } \\
& $\omega_{b}\left[\mathrm{~s}^{-1}\right]$ & $h[\mathrm{~mm}]$ & $d[\mathrm{~mm}]$ & $F[-]$ & $\omega_{b}\left[\mathrm{~s}^{-1}\right]$ & $h[\mathrm{~mm}]$ & $d[\mathrm{~mm}]$ & $F[-]$ \\
\hline Exact & 0.0063 & 0.45 & 1.8 & - & 0.0063 & 1.35 & 2.6 & - \\
\hline Steady-state & 0.0010 & 1.10 & 1.8 & $0.27075 \mathrm{E}-2$ & 0.0066 & 1.30 & 2.7 & $0.33818 \mathrm{E}-2$ \\
& 0.0010 & 1.15 & 1.7 & $0.27129 \mathrm{E}-2$ & 0.0059 & 1.40 & 2.7 & $0.33826 \mathrm{E}-2$ \\
& 0.0010 & 1.05 & 1.9 & $0.27133 \mathrm{E}-2$ & 0.0070 & 1.30 & 2.6 & $0.33832 \mathrm{E}-2$ \\
\hline Transient & 0.0063 & 0.45 & 1.8 & $0.19128 \mathrm{E}+1$ & 0.0063 & 1.35 & 2.6 & $0.18577 \mathrm{E}+1$ \\
& 0.0057 & 0.50 & 1.8 & $0.19178 \mathrm{E}+1$ & 0.0065 & 1.35 & 2.5 & $0.19521 \mathrm{E}+1$ \\
& 0.0060 & 0.50 & 1.7 & $0.19220 \mathrm{E}+1$ & 0.0061 & 1.35 & 2.7 & $0.19621 \mathrm{E}+1$ \\
\hline
\end{tabular}

Table 4: Solution of the steady-state and transient inverse problem using measurement data with $25 \mathrm{mK}$ noise and known metabolic heat generation.

Because the diameter of a pigmented melanoma can be measured easily, let us now assume that we know the metabolic heat generation and diameter of the tumour. Know- 

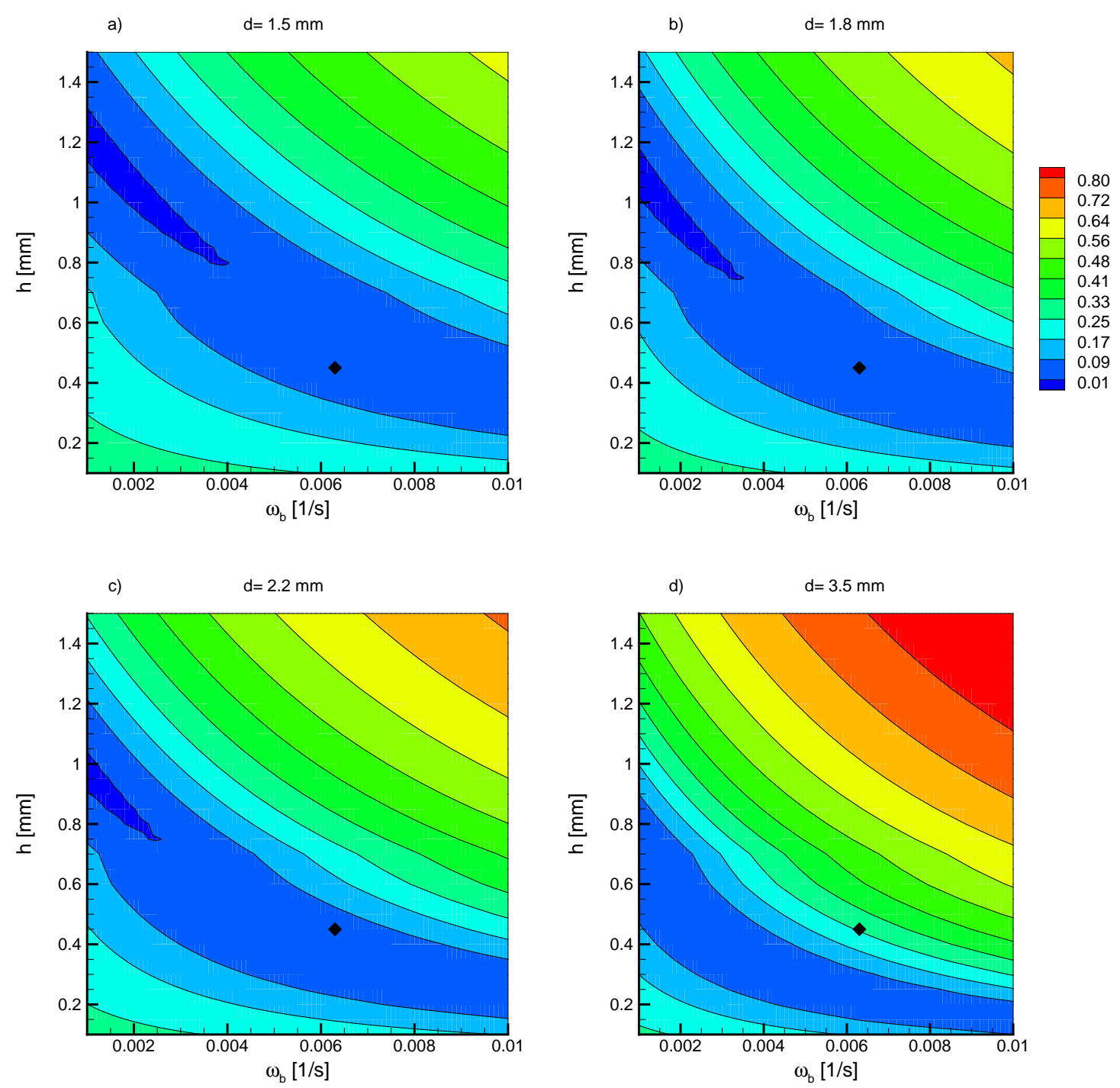

Figure 9: Response surface of objective function for $25 \mathrm{mK}$ noise comparing tumour thickness and blood perfusion rate for Clark II tumour and metabolic heat generation $3700 \mathrm{~W} / \mathrm{m}^{3}$ for different diameters: a) $d=1.5 \mathrm{~mm}$, b) $d=1.8 \mathrm{~mm}$, c) $d=2.2 \mathrm{~mm}$ and d) $d=3.5 \mathrm{~mm}$.

ing these two parameters, we can try to estimate the tumour blood perfusion rate and thickness using static thermography. Tables 6 and 7 show the solution of the steady-state inverse problem for low and high levels of noise. The level of noise compared to the temperature difference between the healthy tissue and the skin tumour under steady-state conditions (Figure 2) seems to be too high even for estimating only two parameters for Clark II size. However, it is possible to estimate the blood perfusion rate and tumour thickness for Clark IV under low measurement noise. For high level of noise the estimation is no longer possible.

We can conclude that it is difficult to estimate several parameters of the tumour simultaneously by using steady-state temperature measurement. It is possible to estimate 

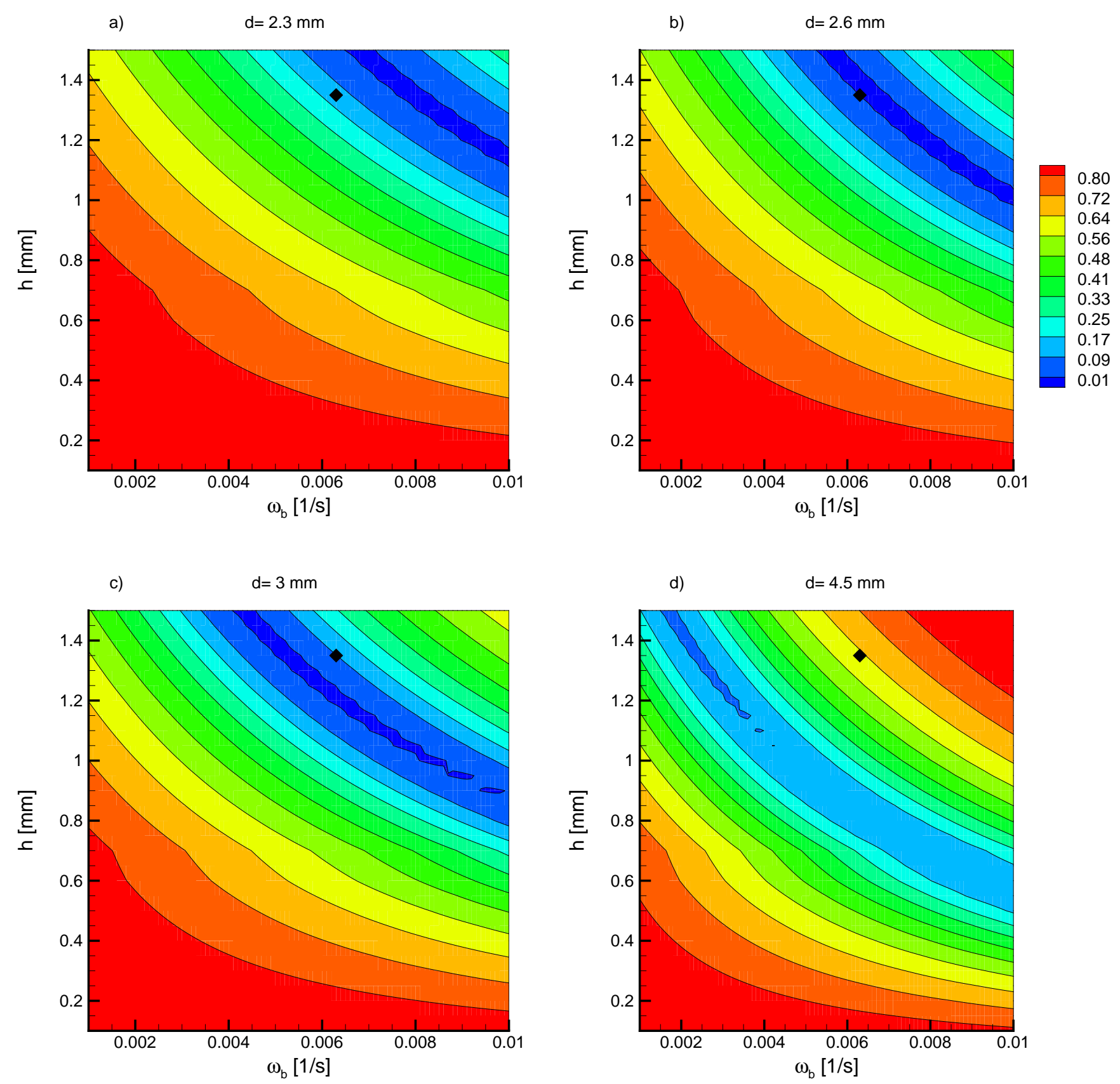

Figure 10: Response surface of objective function for $25 \mathrm{mK}$ noise comparing tumour thickness and blood perfusion rate for Clark IV tumour and metabolic heat generation $3700 \mathrm{~W} / \mathrm{m}^{3}$ for different diameters: a) $d=2.3 \mathrm{~mm}$, b) $d=2.6 \mathrm{~mm}$, c) $d=3.0 \mathrm{~mm}$ and d) $d=4.5 \mathrm{~mm}$.

the diameter, thickness and blood perfusion of the tumour under exact measurement data knowing the metabolic heat generation. However, when we are dealing with measurement noise, which is always present, then the parameters for early stage tumours cannot be retrieved anymore. The parameters can be estimated only for later tumour stages like 


\begin{tabular}{c|cccc|cccc} 
& \multicolumn{5}{|c|}{ Clark II } & \multicolumn{4}{c}{ Clark IV } \\
& $\omega_{b}\left[\mathrm{~s}^{-1}\right]$ & $h[\mathrm{~mm}]$ & $d[\mathrm{~mm}]$ & $F[-]$ & $\omega_{b}\left[\mathrm{~s}^{-1}\right]$ & $h[\mathrm{~mm}]$ & $d[\mathrm{~mm}]$ & $F[-]$ \\
\hline Exact & 0.0063 & 0.45 & 1.8 & - & 0.0063 & 1.35 & 2.6 & - \\
\hline Steady-state & 0.0094 & 0.80 & 0.7 & $0.13126 \mathrm{E}-1$ & 0.031 & 1.50 & 3.1 & $0.94896 \mathrm{E}-2$ \\
& 0.0087 & 0.85 & 0.7 & $0.13127 \mathrm{E}-1$ & 0.0029 & 1.50 & 3.2 & $0.94950 \mathrm{E}-2$ \\
& 0.0098 & 0.60 & 0.9 & $0.13128 \mathrm{E}-1$ & 0.0030 & 1.50 & 3.1 & $0.95092 \mathrm{E}-2$ \\
\hline Transient & 0.0063 & 0.45 & 1.8 & $0.75062 \mathrm{E}+1$ & 0.0063 & 1.35 & 2.6 & $0.75266 \mathrm{E}+1$ \\
& 0.0057 & 0.50 & 1.8 & $0.75102 \mathrm{E}+1$ & 0.0065 & 1.35 & 2.5 & $0.76089 \mathrm{E}+1$ \\
& 0.0067 & 0.40 & 1.9 & $0.75139 \mathrm{E}+1$ & 0.0061 & 1.35 & 2.7 & $0.76478 \mathrm{E}+1$ \\
\hline
\end{tabular}

Table 5: Solution of the steady-state and transient inverse problem using measurement data with $50 \mathrm{mK}$ noise and known metabolic heat generation.

\begin{tabular}{c|ccc|ccc} 
& \multicolumn{3}{|c|}{ Clark II } & \multicolumn{3}{c}{ Clark IV } \\
& $\omega_{b}\left[\mathrm{~s}^{-1}\right]$ & $h[\mathrm{~mm}]$ & $F[-]$ & $\omega_{b}\left[\mathrm{~s}^{-1}\right]$ & $h[\mathrm{~mm}]$ & $F[-]$ \\
\hline Exact & 0.0063 & 0.45 & - & 0.0063 & 1.35 & - \\
\hline Steady-state & 0.0010 & 1.10 & $0.27075 \mathrm{E}-2$ & 0.0070 & 1.30 & $0.33832 \mathrm{E}-2$ \\
& 0.0010 & 1.05 & $0.28033 \mathrm{E}-2$ & 0.0066 & 1.35 & $0.33842 \mathrm{E}-2$ \\
& 0.0010 & 1.00 & $0.30078 \mathrm{E}-2$ & 0.0063 & 1.35 & $0.33877 \mathrm{E}-2$ \\
\hline Transient & 0.0063 & 0.45 & $0.19128 \mathrm{E}+1$ & 0.0063 & 1.35 & $0.18577 \mathrm{E}+1$ \\
& 0.0057 & 0.50 & $0.19178 \mathrm{E}+1$ & 0.0061 & 1.40 & $0.20880 \mathrm{E}+1$ \\
& 0.0052 & 0.55 & $0.19349 \mathrm{E}+1$ & 0.0060 & 1.45 & $0.24232 \mathrm{E}+1$ \\
\hline
\end{tabular}

Table 6: Solution of the steady-state and transient inverse problem for known metabolic heat generation and tumour diameter using measurement data with $25 \mathrm{mK}$ noise.

\begin{tabular}{c|ccc|ccc} 
& \multicolumn{3}{|c|}{ Clark II } & \multicolumn{3}{c}{ Clark IV } \\
& $\omega_{b}\left[\mathrm{~s}^{-1}\right]$ & $h[\mathrm{~mm}]$ & $F[-]$ & $\omega_{b}\left[\mathrm{~s}^{-1}\right]$ & $h[\mathrm{~mm}]$ & $F[-]$ \\
\hline Exact & 0.0063 & 0.45 & - & 0.0063 & 1.35 & - \\
\hline Steady-state & 0.0094 & 0.30 & $0.13275 \mathrm{E}-1$ & 0.047 & 1.45 & $0.95968 \mathrm{E}-2$ \\
& 0.0094 & 0.30 & $0.13246 \mathrm{E}-1$ & 0.0044 & 1.50 & $0.95974 \mathrm{E}-2$ \\
& 0.0078 & 0.35 & $0.13247 \mathrm{E}-1$ & 0.0043 & 1.50 & $0.90530 \mathrm{E}-2$ \\
\hline Transient & 0.0063 & 0.45 & $0.75061 \mathrm{E}+1$ & 0.0063 & 1.35 & $0.75266 \mathrm{E}+1$ \\
& 0.0057 & 0.50 & $0.75102 \mathrm{E}+1$ & 0.0061 & 1.40 & $0.77692 \mathrm{E}+1$ \\
& 0.0052 & 0.55 & $0.75258 \mathrm{E}+1$ & 0.0060 & 1.45 & $0.80819 \mathrm{E}+1$ \\
\hline
\end{tabular}

Table 7: Solution of the steady-state and transient inverse problem for known metabolic heat generation and tumour diameter using measurement data with $50 \mathrm{mK}$ noise.

Clark IV under low measurement noise.

\subsection{Transient results}

Similar to the steady-state inverse problem, the sensitivity of the metabolic heat generation is small and it will be difficult to estimate it. The response surface using exact 
measurement data according to a constant diameter and several different tumour thicknesses, comparing metabolic heat generation and blood perfusion rate, can be seen in figure 11 for Clark II size.
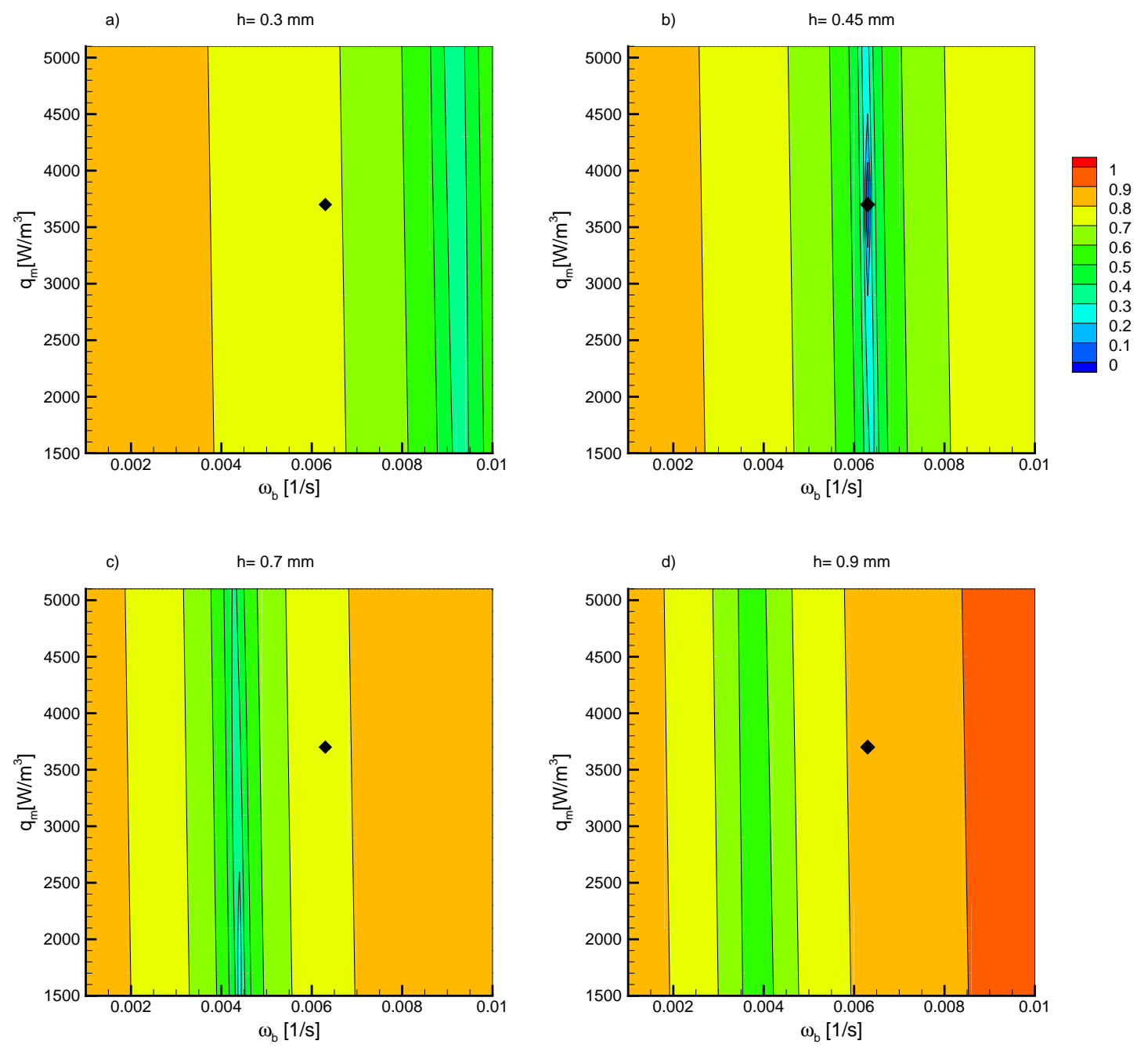

Figure 11: Response surface of objective function for exact measurement data comparing metabolic heat generation and blood perfusion for Clark II tumour at exact diameter $1.8 \mathrm{~mm}$ and different tumour thicknesses: a) $h=0.3 \mathrm{~mm}$, b) $h=0.45 \mathrm{~mm}$, c) $h=0.7 \mathrm{~mm}$ and d) $h=0.9 \mathrm{~mm}$.

Therefore, for our further inverse analyses, we will be focusing on evaluation and sensitivity of the other three parameters, fixing the metabolic heat generation. Figure 12 shows the response surface of the objective function for exact measurement data correlating the blood perfusion rate and tumour thickness for several different diameters at exact metabolic heat generation for Clark II size, while figure 13 shows the same correlation for Clark IV size. As can be seen from both figures, the correlation between these three parameters is much stronger compared to the steady-state problem (figures 7 and 8). In the transient inverse problem, there is no distinct band of low objective function values that would indicate multiple solutions. The plot for the exact tumour diameter 
shows a distinctive location of the global minimum, which is even better expressed for the Clark IV tumour, unfortunately covered with the dot, which represents the exact solution. From the higher parameter sensitivity, we can conclude that all three parameters can be estimated more easily, and also that measurement noise would not have a large influence on the solution. Table 2 shows the three best obtained solutions using transient inverse problem with corresponding value of the objective function for exact measurement data. We can observe that the estimated parameters for Clark II and Clark IV are still within a satisfactory range of accuracy, and that the best solution coincides with the exact one. We were able to find the exact solution as for the steady-state case, while the other best solutions for Clark II are much closer to the exact one using transient measurement data compared to the steady-state one. However, if we do not know the exact metabolic heat generation, we can still estimate all three parameters quite accurately, as can be seen in table 3. We should state here that the minimum value of the objective function is different between the steady-state and transient cases and cannot be compared, because of the different number of measurement data as already discussed.

Under noisy measurement data, figure 14 shows the response surface for a Clark II tumour, using $25 \mathrm{mK}$ noise, correlating blood perfusion rate and tumour thickness for known metabolic heat generation, while figure 15 shows the same for Clark IV. From both figures, we can observe the region of low objective function values corresponding to the exact solution, especially for the exact tumour diameter. This region is larger for the smaller tumour size, which indicates that we can obtain multiple solutions with similar values of the objective function. If the estimated blood perfusion is lower then the estimated thickness will be higher. However, the region for Clark IV is small indicating that parameters can be estimated more easily. With the increase of noise, these regions become wider but still close to the exact solution as can be seen in figures 16 and 17 for Clark II and Clark IV, respectively. Tables 4 and 5 show the obtained solutions for noisy measurement data. We can observe that it is possible to estimate the exact solutions for Clark II and Clark IV test cases, even for high noise level. The best solution is the same as the exact one, while the other two best solutions are near. This shows the advantage of the transient over the steady-state measurement approach. Similar results are found for the case when the metabolic heat generation and tumour diameter are known. For this situation, we can obtain the exact solution for blood perfusion rate and tumour thickness for both test cases under low and high levels of measurement noise, as can be seen from tables 6 and 7 .

Table 8 shows the best obtained solutions for the transient inverse problem under noisy measurement data when the metabolic heat generation is predetermined or estimated. As we can observe, the value of the metabolic heat generation does not have a great impact on the other estimated parameters, regardless of noise and especially for the Clark IV tumour size. This means that blood perfusion rate, the diameter and the thickness of the tumour could be easily obtained even if the metabolic heat generation is not known exactly.

Table 9 shows the best obtained solutions by DOE for steady-state and transient inverse problems in the investigated space for noisy measurement data not imposing any constraints. This represents the feasibility of estimating all four parameters for Clark II and Clark IV tumours using static or dynamic thermography. As can be seen from table 9, the static measurements can only estimate Clark IV tumour parameters under low measurement noise, excluding the metabolic heat generation. Using a dynamic 

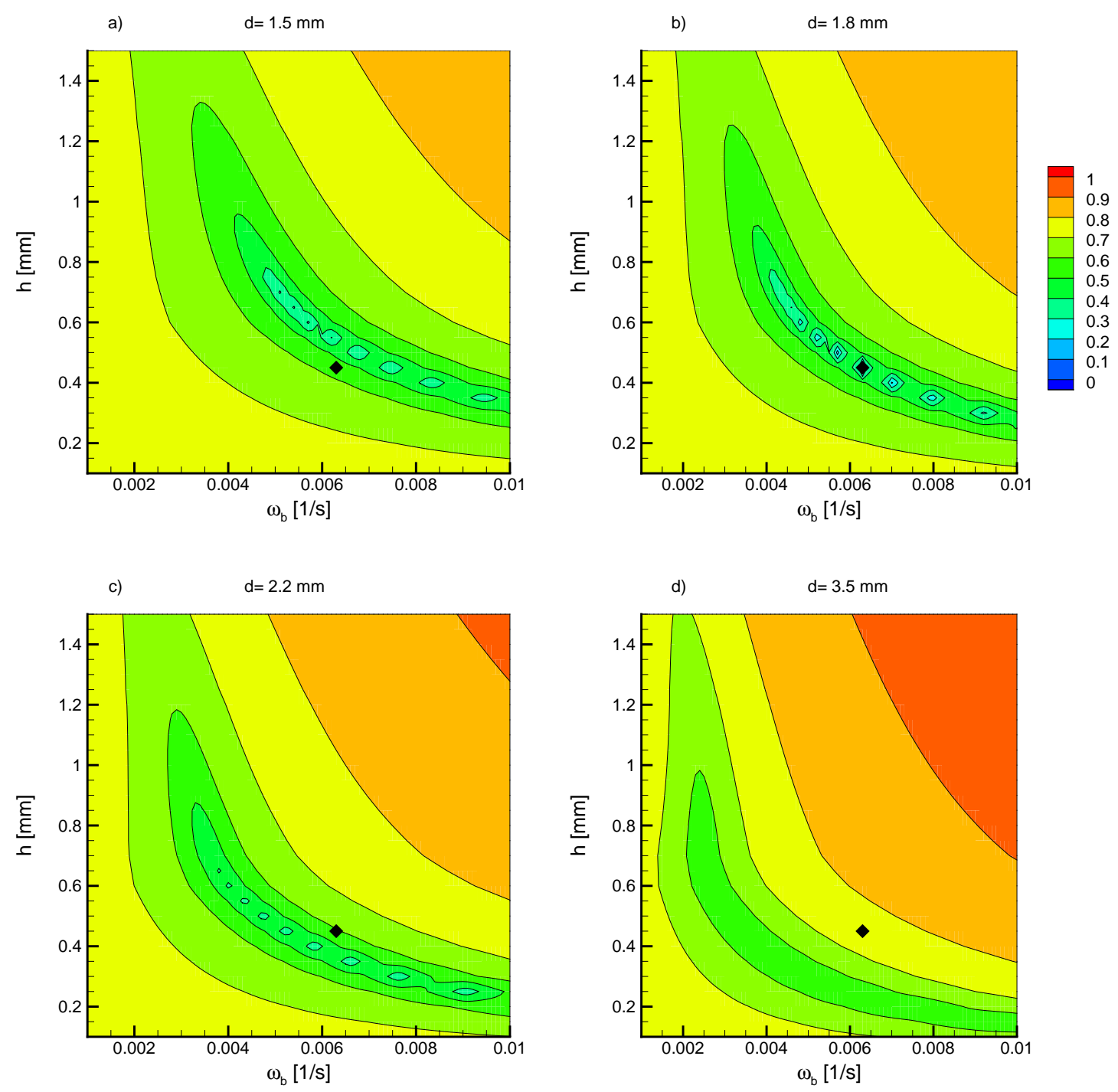

Figure 12: Response surface of objective function for exact measurement data comparing blood perfusion rate and tumour thickness for Clark II tumour at metabolic heat generation $q_{m}=3700 \mathrm{~W} / \mathrm{m}^{3}$ and different diameters: a) $d=1.5 \mathrm{~mm}$, b) $d=1.8 \mathrm{~mm}$, c) $d=2.2 \mathrm{~mm}$ and d) $d=3.5 \mathrm{~mm}$.

measurement technique, it is possible to estimate all four parameters for Clark II and Clark IV tumours, regardless of noise. This clearly demonstrates the superior efficiency of dynamic measurements over static ones. Despite the low sensitivity of the metabolic heat generation, it is still possible to determine this parameter under noisy measurement data. This is also visible from table 8 , where we can observe the minimum values of the objective function for the exact metabolic heat rate. 

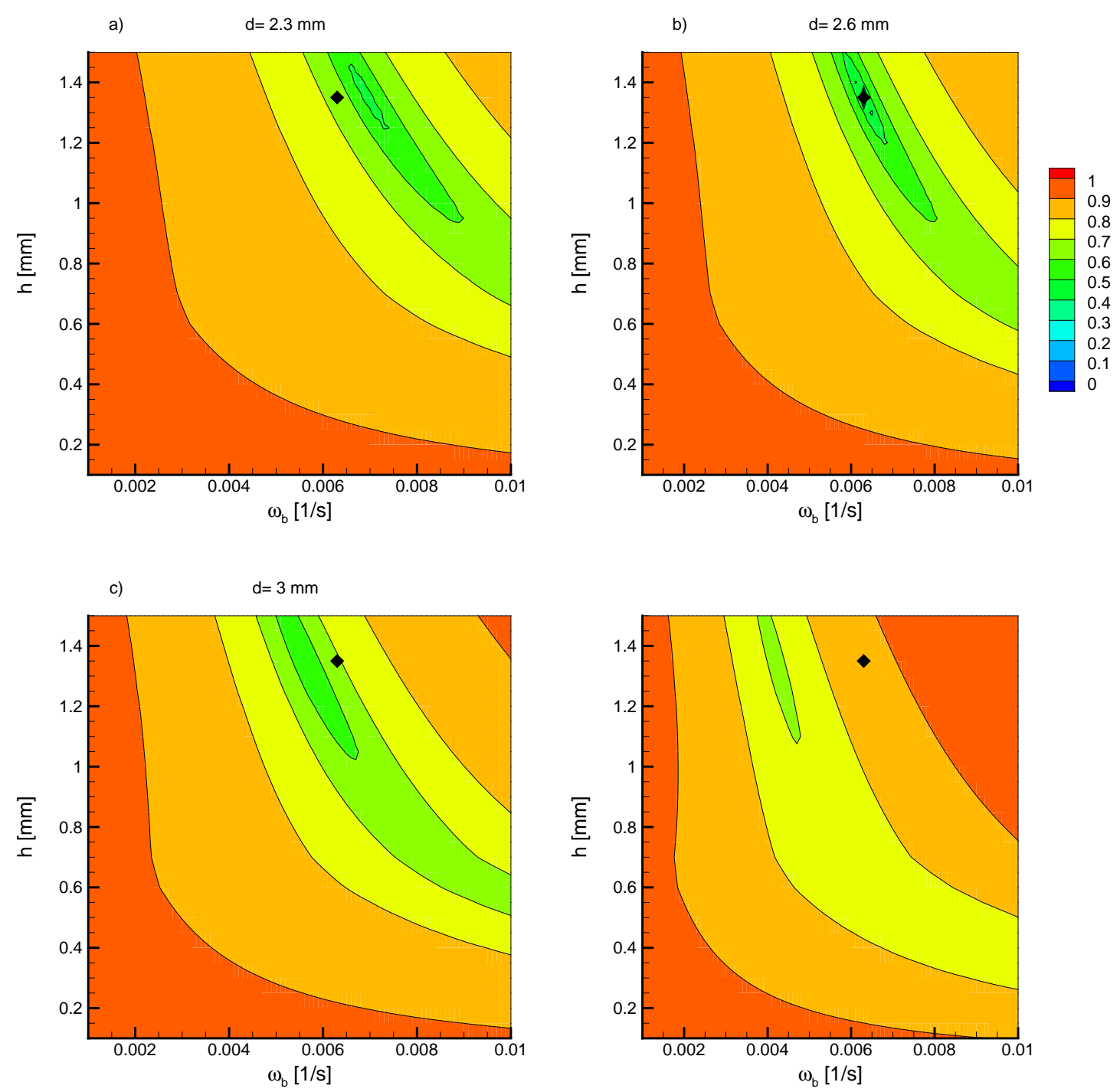

Figure 13: Response surface of objective function for exact measurement data comparing blood perfusion rate and tumour thickness for Clark IV tumour at metabolic heat generation $q_{m}=3700 \mathrm{~W} / \mathrm{m}^{3}$ and different diameters: a) $d=2.3 \mathrm{~mm}$, b) $d=2.6 \mathrm{~mm}$, c) $d=3.0 \mathrm{~mm}$ and d) $d=4.5 \mathrm{~mm}$.

\section{Conclusion}

This paper discusses numerical techniques for two different inverse bioheat problems, to estimate skin tumour parameters. The first uses steady-state skin temperature measurements reflecting static thermography, while the second one employs transient measurements used in dynamic thermography. The aim is to investigate which of these two approaches is more suitable for detecting skin tumour in its early stage, and also to contribute to the development of new non-invasive diagnostic techniques using IR imaging.

The most important skin tumour parameter for dermatologists is the tumour thickness, which reflects the stage and progression of the tumour. However, in this paper, we try to estimate not only tumour thickness but also diameter, blood perfusion rate and 

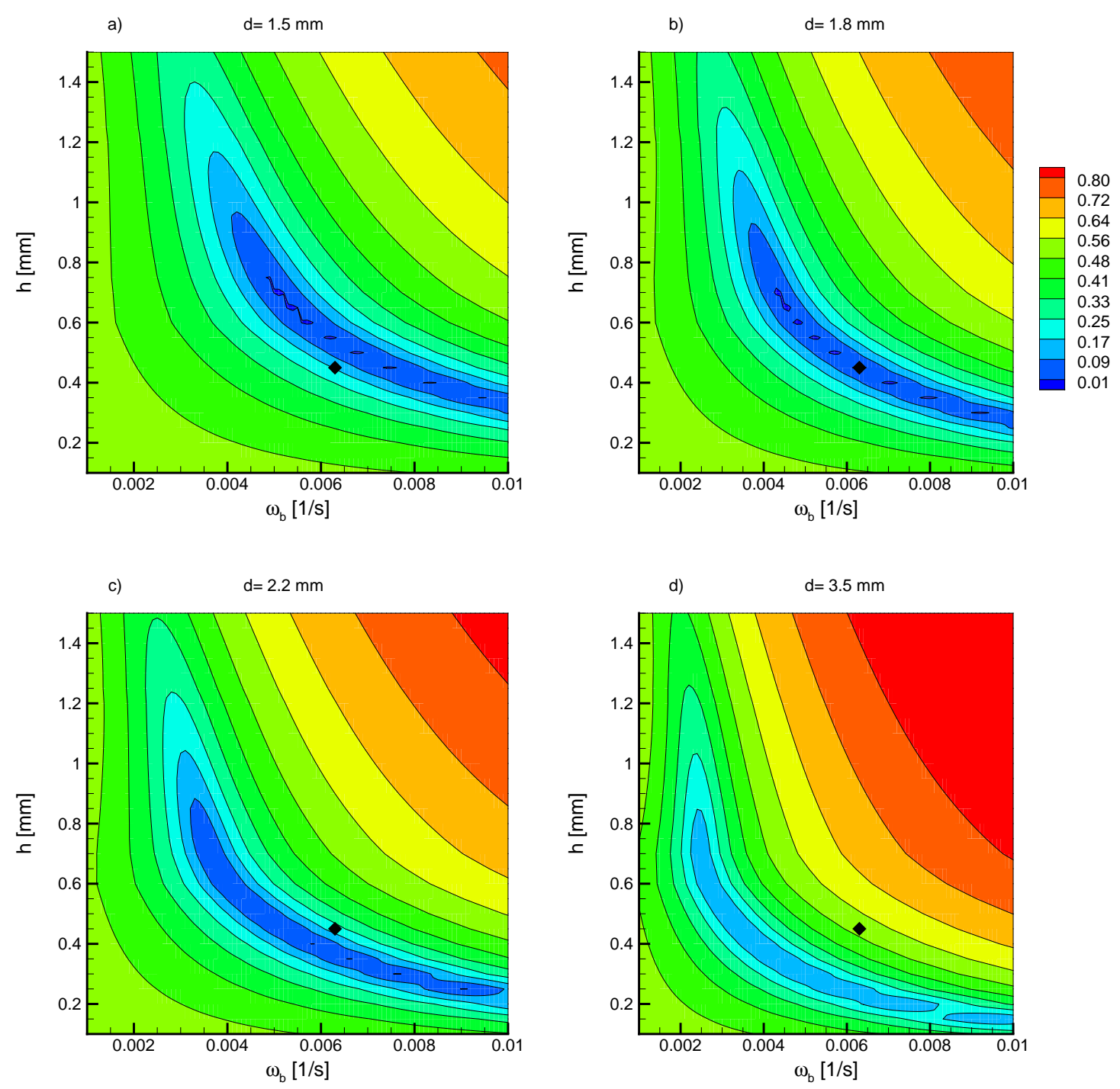

Figure 14: Response surface of objective function for measurement data with $25 \mathrm{mK}$ noise comparing blood perfusion rate and tumour thickness for Clark II tumour at metabolic heat generation $q_{m}=3700 \mathrm{~W} / \mathrm{m}^{3}$ and different diameters: a) $d=1.5 \mathrm{~mm}$, b) $d=1.8 \mathrm{~mm}$, c) $d=2.2 \mathrm{~mm}$ and d) $d=3.5 \mathrm{~mm}$.

metabolic heat generation. The last two parameters could reflect the speed of tumour progression. To estimate tumour parameters based on the measured skin surface temperature, we have to solve an inverse problem, which is based on the numerical bioheat model. For this step we used a numerical model based on Pennes bioheat equation and a multilayer tissue composed of several different layers and tumour considering constant material properties. For now, we neglect some of the mechanisms like temperature change of thermal properties and blood perfusion rate in the cooling-rewarming process, which would be difficult to model mathematically and hard to validate. With the proposed numerical model, we can still evaluate the tumour parameters, however in an average sense. The success of parameter estimation lies in the accuracy of the numerical model 

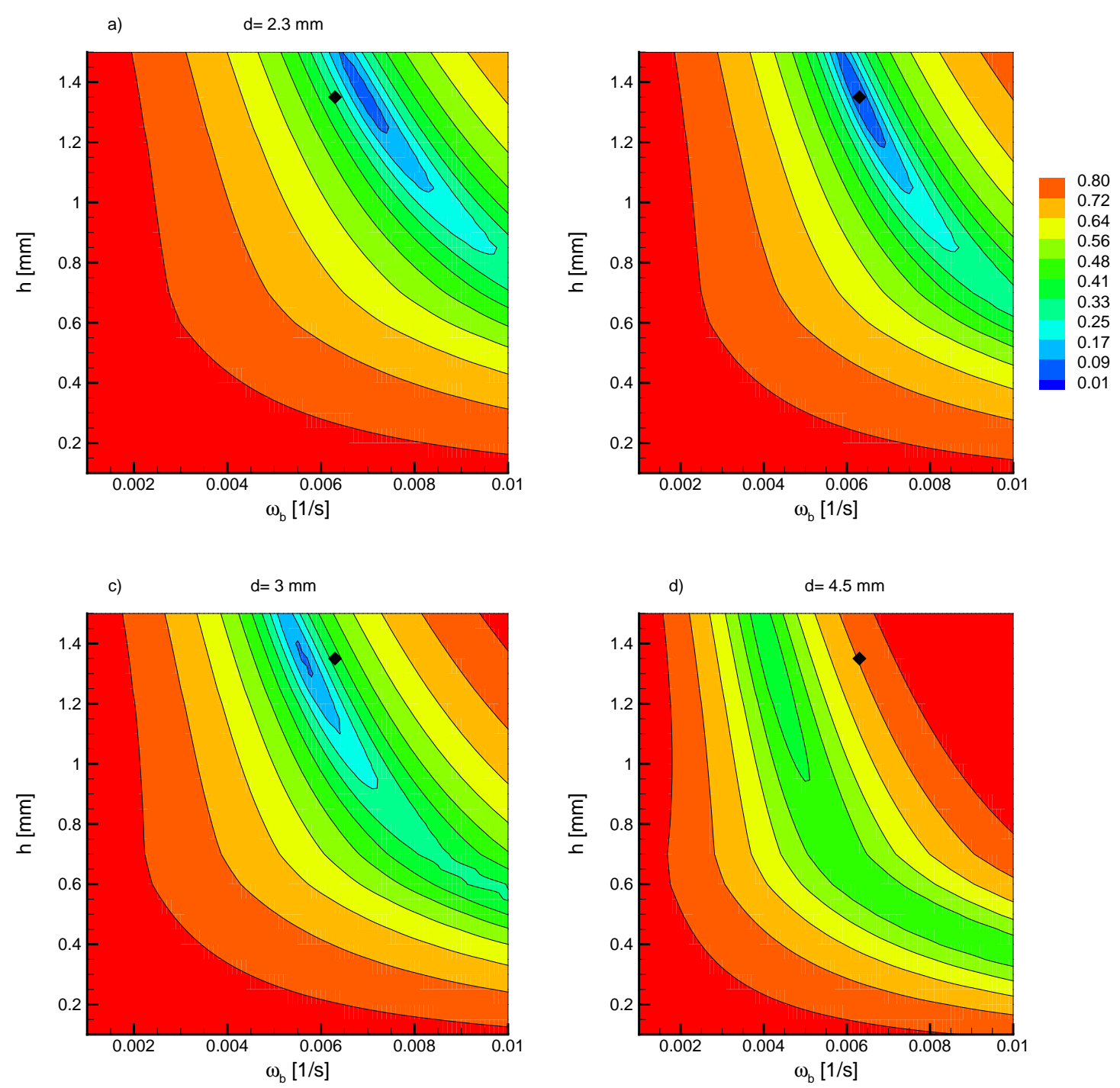

Figure 15: Response surface of objective function for measurement data with $25 \mathrm{mK}$ noise comparing blood perfusion rate and tumour thickness for Clark IV tumour at metabolic heat generation $q_{m}=3700 \mathrm{~W} / \mathrm{m}^{3}$ and different diameters: a) $d=2.3 \mathrm{~mm}$, b) $d=2.6 \mathrm{~mm}$, c) $d=3.0 \mathrm{~mm}$ and d) $d=4.5 \mathrm{~mm}$.

and the measurement noise. To test the inverse problems we generated two test examples which differ in tumour size (Clark II and Clark IV), which have been used to numerically generate the measurement data with and without noise. For inverse problem solving, the general full factorial DOE approach has been used which was helpful in making the analysis of the solution space and to observe the response surface of the objective function. For this 2, 306, 486 simulations were made for each inverse problem.

The results shows that all four parameters could be determined simultaneously under noisy measurement data only for the transient inverse problem, which shows an advantage over the steady-state measurement. The analyses of the design space show that the sensitivity of the metabolic heat generation is small, so it would be hard to estimate it 

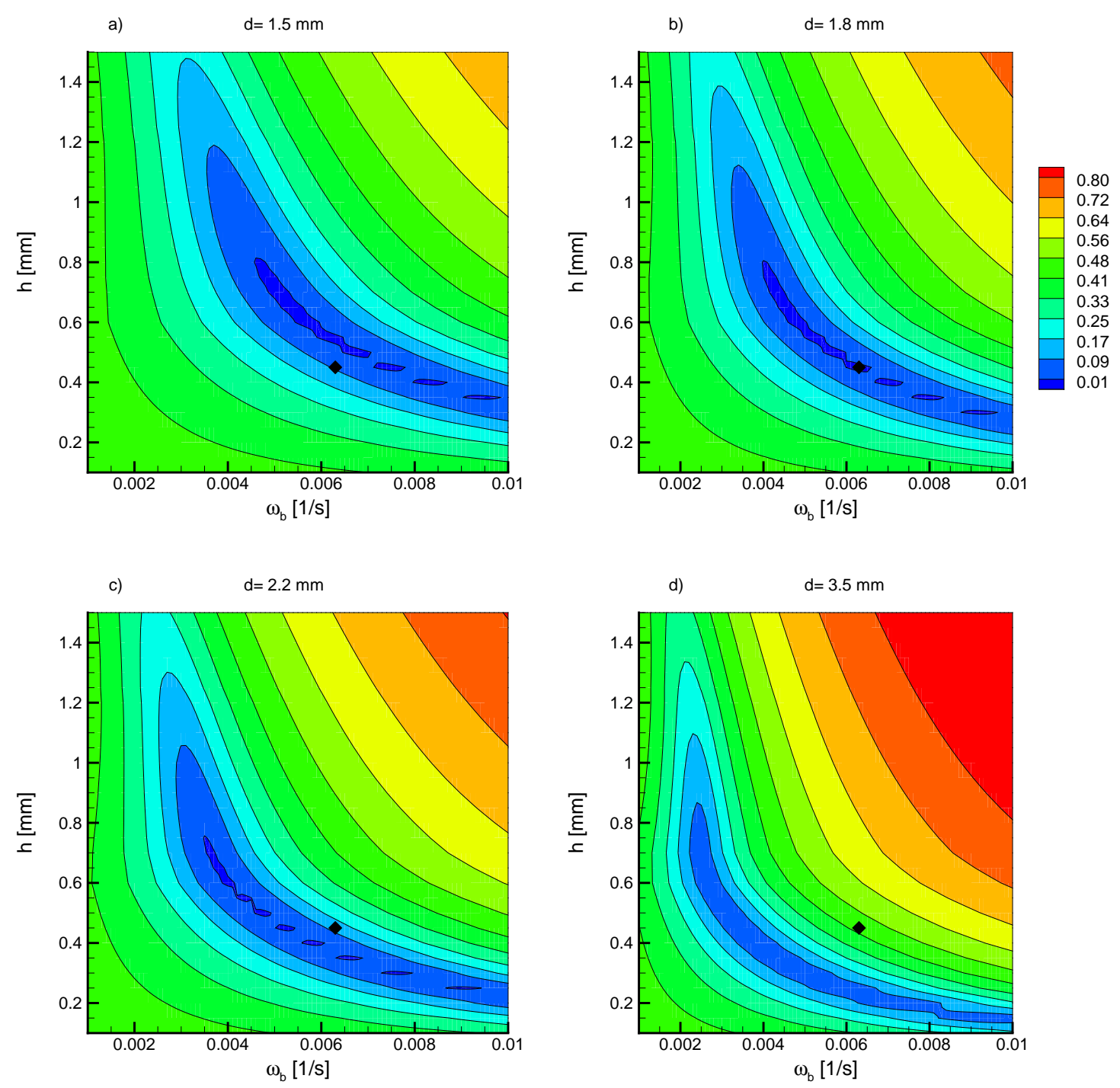

Figure 16: Response surface of objective function for measurement data with $50 \mathrm{mK}$ noise comparing blood perfusion rate and tumour thickness for Clark II tumour at metabolic heat generation $q_{m}=3700 \mathrm{~W} / \mathrm{m}^{3}$ and different diameters: a) $d=1.5 \mathrm{~mm}$, b) $d=1.8 \mathrm{~mm}$, c) $d=2.2 \mathrm{~mm}$ and d) $d=3.5 \mathrm{~mm}$.

using gradient based optimization, especially for noisy measurement data. However, fixing or estimating the metabolic heat generation showed that the other three parameters are well correlated with higher sensitivity, which shows the possibility of estimating all three parameters even for the steady-state case. Unfortunately, for steady-state measurement data, this can be done only for exact data or low level of noise and advanced tumour. The temperature difference between the healthy tissue and early tumour stage under steady-state conditions is very small, and the measurement error overrides this difference making the estimation of parameters very hard, even when fixing the metabolic heat generation and diameter of the tumor. However, using a cooling-rewarming technique, the estimation of parameters is much more feasible for Clark II and Clark IV tumours, 

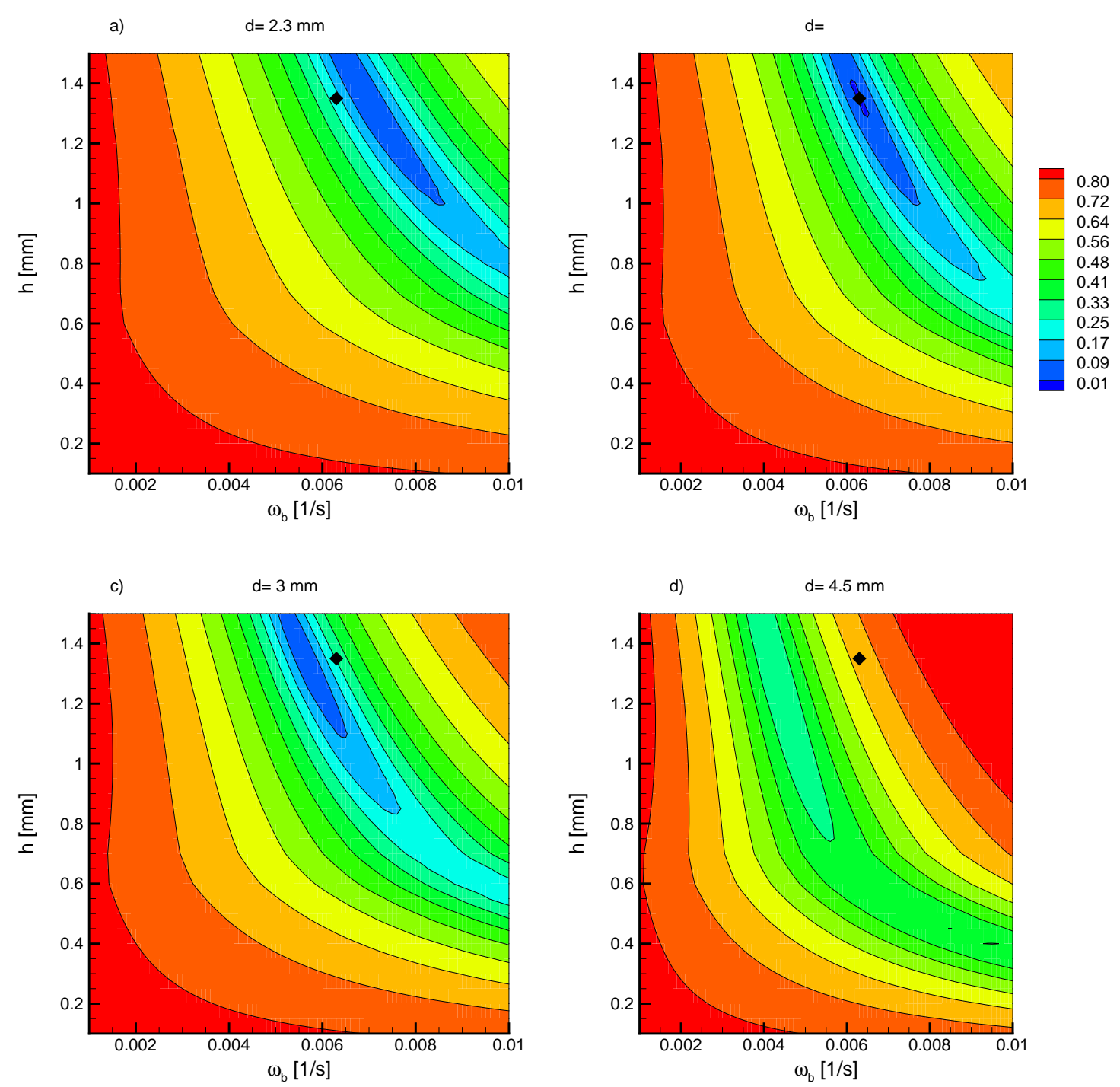

Figure 17: Response surface of objective function for measurement data with $50 \mathrm{mK}$ noise comparing blood perfusion rate and tumour thickness for Clark IV tumour at metabolic heat generation $q_{m}=3700 \mathrm{~W} / \mathrm{m}^{3}$ and different diameters: a) $d=2.3 \mathrm{~mm}$, b) $d=2.6 \mathrm{~mm}$, c) $d=3.0 \mathrm{~mm}$ and d) $d=4.5 \mathrm{~mm}$.

even for high level of noise. The results and analysis showed that the dynamic technique is superior to the static thermography, and that it is possible to detect the tumour in its early stage.

The analysis of both inverse problems also showed that the objective function is smooth and thus gradient optimization algorithms (such as the Levenberg-Marquardt method) can be used for identification of tumour diameter, thickness and blood perfusion, to speed up the computational time. However, due to the low sensitivity of the metabolic heat generation, it is advised to use a stochastic optimization technique like GA or DOE. Because of the smooth model response it would be also possible to use SM on the RCBD DOE, which would speed up the optimization process and make the algorithm more 


\begin{tabular}{c|cccc|cccc} 
& \multicolumn{5}{|c|}{ Clark II } & \multicolumn{4}{c}{ Clark IV } \\
& $\omega_{b}\left[s^{-1}\right]$ & $h[\mathrm{~mm}]$ & $d[\mathrm{~mm}]$ & $F[-]$ & $\omega_{b}\left[\mathrm{~s}^{-1}\right]$ & $h[\mathrm{~mm}]$ & $d[\mathrm{~mm}]$ & $F[-]$ \\
\hline Exact & 0.0063 & 0.45 & 1.8 & - & 0.0063 & 1.35 & 2.6 \\
\hline$q_{m}\left[W / m^{3}\right]$ & \multicolumn{5}{|c|}{$25 m K$ noise } & \multicolumn{4}{c}{$25 m K$ noise } \\
\hline 2700 & 0.0051 & 0.60 & 1.7 & $0.19216 \mathrm{E}+1$ & 0.0063 & 1.35 & 2.6 & $0.19952 \mathrm{E}+1$ \\
3700 & 0.0063 & 0.45 & 1.8 & $0.19128 \mathrm{E}+1$ & 0.0063 & 1.35 & 2.6 & $0.18577 \mathrm{E}+1$ \\
4500 & 0.0070 & 0.40 & 1.8 & $0.19142 \mathrm{E}+1$ & 0.0064 & 1.35 & 2.6 & $0.19414 \mathrm{E}+1$ \\
\hline$q_{m}\left[W / m^{3}\right]$ & \multicolumn{9}{|c|}{$50 \mathrm{mK}$ noise } & & \multicolumn{5}{c}{$50 \mathrm{mK}$ noise } \\
\hline 2700 & 0.0063 & 0.45 & 1.8 & $0.75122 \mathrm{E}+1$ & 0.0063 & 1.35 & 2.6 & $0.76695 \mathrm{E}+1$ \\
3700 & 0.0063 & 0.45 & 1.8 & $0.75062 \mathrm{E}+1$ & 0.0063 & 1.35 & 2.6 & $0.75266 \mathrm{E}+1$ \\
4500 & 0.0070 & 0.40 & 1.8 & $0.75089 \mathrm{E}+1$ & 0.0063 & 1.35 & 2.6 & $0.76059 \mathrm{E}+1$ \\
\hline
\end{tabular}

Table 8: Solution of the transient inverse problem for different metabolic heat generation values using noisy measurement data.

\begin{tabular}{c|cccc|cccc} 
& \multicolumn{5}{|c|}{ Clark II } & \multicolumn{5}{c}{ Clark IV } \\
& $\omega_{b}\left[s^{-1}\right]$ & $q_{m}\left[W / m^{3}\right]$ & $h[\mathrm{~mm}]$ & $d[\mathrm{~mm}]$ & $\omega_{b}\left[\mathrm{~s}^{-1}\right]$ & $q_{m}\left[\mathrm{~W} / \mathrm{m}^{3}\right]$ & $h[\mathrm{~mm}]$ & $d[\mathrm{~mm}]$ \\
\hline Exact & 0.0063 & 3700 & 0.45 & 1.8 & 0.0063 & 3700 & 1.35 & 2.6 \\
\hline & \multicolumn{7}{|c}{ Steady-state } \\
\hline $25 m K$ & 0.0010 & 1500 & 1.20 & 2.0 & 0.0062 & 4500 & 1.30 & 2.7 \\
$50 m K$ & 0.0010 & 5100 & 0.55 & 0.9 & 0.0032 & 3500 & 1.50 & 3.1 \\
\hline & \multicolumn{7}{|c}{ Transient } \\
\hline $25 m K$ & 0.0063 & 3700 & 0.45 & 1.8 & 0.0063 & 3700 & 1.35 & 2.6 \\
$50 m K$ & 0.0063 & 3500 & 0.45 & 1.8 & 0.0063 & 3700 & 1.35 & 2.6 \\
\hline
\end{tabular}

Table 9: Solution of the steady-state and transient inverse problem for all four parameters using noisy measurement data.

acceptable for practical implementation. The full factorial DOE used in this paper is only attractive from the research point of view because of the obtained model sensitivity and the design space investigation in exchange to high computational costs.

This paper demonstrates that it is possible to detect several tumour parameters using a dynamic approach, which is important for the development of new diagnostic tools and also for the monitoring of the progression of the disease. However, for clinical implementation, it is important that the method is accurate and fast. The numerical model based upon which the parameters are evaluated should be mimicking the thermal and pathological behaviour of the skin tumour and surrounding tissue as realistically as possible. In future, we plan to develop a more appropriate 3D numerical model that would include the temperature variation of the blood perfusion rate, which is in our opinion the most important mechanism here. We will also be testing several different optimization algorithms to speed up the computational time, and will also focus on the boundary conditions for the model and their influence on the accuracy of the estimated parameters. There are also other practical obstacles, for example how to assure the constant temperature of the skin during the cooling process or how to evaluate the amount of cold applied to the skin. For this reason, this paper represents the base for further investigation and research of 
dynamic thermography for early skin cancer detection.

\section{References}

[1] F. Greene, C. Compton, A. Fritz, J. Shah, D. Winchester, Melanoma of the skin, AJCC Cancer Staging Atlas Part V (2006).

[2] W. H. Clark, L. From, E. A. Bernardino, M. C. Mihm, The histogenesis and biologic behavior of primary human malignant melanomas of the skin, Cancer Research 29 (3) (1969) 705-727.

[3] A. Breslow, Thickness, cross-sectional areas and depth of invasion in the prognosis of cutaneous melanoma., Annals of Surgery 172 (5) (1970) 902-908.

[4] J. E. Mayer, S. M. Swetter, T. Fu, A. C. Geller, Screening, early detection, education, and trends for melanoma: current status (2007-2013) and future directions: Part I. Epidemiology, high-risk groups, clinical strategies, and diagnostic technology, Journal of the American Academy of Dermatology 71 (4) (2014) 599.e1-599.e12.

[5] J. E. Mayer, S. M. Swetter, T. Fu, A. C. Geller, Screening, early detection, education, and trends for melanoma: current status (2007-2013) and future directions: Part II. Screening, education, and future directions, Journal of the American Academy of Dermatology 71 (4) (2014) 611.e1-611.e10.

[6] D. Wartman, M. Weinstock, Are we overemphasizing sun avoidance in protection from melanoma?, Cancer Epidemiology and Prevention Biomarkers 17 (3) (2008) 469-470.

[7] D. S. Rigel, J. Russak, R. Friedman, The evolution of melanoma diagnosis: 25 years beyond the ABCDs, CA Cancer J. Clin. 60 (5) (2010) 301-316.

[8] R. Marchesini, A. Bono, C. Bartoli, M. Lualdi, S. Tomatis, N. Cascinelli, Optical imaging and automated melanoma detection: questions and answers, Melanoma Research 12 (3) (2002) 279-286.

[9] A. A. Marghoob, L. D. Swindle, C. Z. Moricz, F. A. S. Negron, B. Slue, A. C. Halpern, A. W. Kopf, Instruments and new technologies for the in-vivo diagnosis of melanoma, Journal of the American Academy of Dermatology 49 (5) (2003) 777-797.

[10] E. L. Psaty, A. C. Halpern, Current and emerging technologies in melanoma diagnosis: the state of the art, Clinics in Dermatology 27 (1) (2009) 35-45.

[11] S. Q. Wang, H. Rabinovitz, A. W. Kopf, M. Oliviero, Current technologies for the in vivo diagnosis of cutaneous melanomas, Clinics in Dermatology 22 (3) (2004) $217-222$.

[12] J. K. Patel, S. Konda, O. A. Perez, S. Amini, G. Elgart, B. Berman, Newer technologies/techniques and tools in the diagnosis of melanoma, European Journal of Dermatology 18 (6) (2008) 617-631. 
[13] D. Gutkowicz-Krusin, M. Elbaum, A. Jacobs, S. Keem, A. Kopf, H. Kamino, S. Wang, P. Rubin, H. Rabinovitz, M. Oliviero, Precision of automatic measurements of pigmented skin lesion parameters with a MelaFind(TM) multispectral digital dermoscope, Melanoma Research 10 (6) (2000) 563-570.

[14] A. Bhowmik, R. Repaka, S. C. Mishra, K. Mitra, Analysis of radiative signals from normal and malignant human skins subjected to a short-pulse laser, International Journal of Heat and Mass Transfer 68 (2014) 278-294.

[15] J. March, M. Hand, D. Grossman, Practical application of new technologies for melanoma diagnosis: Part i. noninvasve approaches, Journal of the American Academy of Dermatology 72 (6) (2015) 929-941.

[16] L. Lim, B. Nichols, M. R. Migden, N. Rajaram, J. S. Reichenberg, M. K. Markey, M. I. Ross, J. W. Tunnell, Clinical study of noninvasive in vivo melanoma and nonmelanoma skin cancers using multimodal spectral diagnosis, Journal of Biomedical Optics 19 (11) (2014) 117003.

[17] G. Shi, F. Han, C. Liang, L. Wang, K. Li, A novel method of thermal tomography tumor diagnosis and its clinical practice, Applied Thermal Engineering 73 (1) (2014) 408-415.

[18] J. F. Head, F. Wang, C. A. Lipari, R. L. Elliott, The important role of infrared imaging in breast cancer, IEEE Engineering in Medicine and Biology Magazine 19 (3) (2000) 52-57.

[19] A. Di Carlo, Thermography and the possibilities for its applications in clinical and experimental dermatology, Clinics in Dermatology 13 (4) (1995) 329-336.

[20] T. M. Buzug, S. Schumann, L. Pfaffmann, U. Reinhold, J. Ruhlmann, Functional infrared imaging for skin-cancer screening, in: Engineering in Medicine and Biology Society, 2006. EMBS'06. 28th Annual International Conference of the IEEE, IEEE, 2006, pp. 2766-2769.

[21] H. Wang, D. R. Wade Jr, J. Kam, Ir imaging of blood circulation of patients with vascular disease, in: Defense and Security, International Society for Optics and Photonics, 2004, pp. 115-123.

[22] G. Santa Cruz, J. Bertotti, J. Marin, S. Gonzalez, S. Gossio, D. Alvarez, B. Roth, P. Menéndez, M. Pereira, M. Albero, et al., Dynamic infrared imaging of cutaneous melanoma and normal skin in patients treated with BNCT, Applied Radiation and Isotopes 67 (7) (2009) S54-S58.

[23] A. Helmy, M. Holdmann, M. Rizkalla, Application of thermography for non-invasive diagnosis of thyroid gland disease, IEEE Transactions on Biomedical Engineering 55 (3) (2008) 1168-1175.

[24] J.-H. Tan, E. Ng, U. R. Acharya, C. Chee, Infrared thermography on ocular surface temperature: a review, Infrared Physics \& Technology 52 (4) (2009) 97-108. 
[25] A. L. Shada, L. T. Dengel, G. R. Petroni, M. E. Smolkin, S. Acton, C. L. Slingluff, Infrared thermography of cutaneous melanoma metastases, Journal of Surgical Research 182 (1) (2013) e9-e14.

[26] M. P. Çetingül, C. Herman, Quantification of the thermal signature of a melanoma lesion, International Journal of Thermal Sciences 50 (4) (2011) 421-431.

[27] M. Bonmarin, F.-A. Le Gal, Lock-in thermal imaging for the early-stage detection of cutaneous melanoma: A feasibility study, Computers in Biology and Medicine 47 (2014) 36-43.

[28] J. R. Keyserlingk, P. Ahlgren, E. Yu, N. Belliveau, M. Yassa, Functional infrared imaging of the breast: Historical perspectives, current application and future considerations, CRC Press, 2006.

[29] B. Lahiri, S. Bagavathiappan, T. Jayakumar, J. Philip, Medical applications of infrared thermography: a review, Infrared Physics \& Technology 55 (4) (2012) 221-235.

[30] R. Hatwar, C. Herman, Inverse method for quantitative characterisation of breast tumours from surface temperature data, International Journal of Hyperthermia 33 (7) (2017) 1-17.

[31] K. Ammer, E. Ring, Standard procedures for infrared imaging in medicine, CRC Press, 2006.

[32] T.-Y. Cheng, C. Herman, Analysis of skin cooling for quantitative dynamic infrared imaging of near-surface lesions, International Journal of Thermal Sciences 86 (2014) $175-188$.

[33] M. Paruch, E. Majchrzak, Identification of tumor region parameters using evolutionary algorithm and multiple reciprocity boundary element method, Engineering Applications of Artificial Intelligence 20 (5) (2007) 647-655.

[34] P. W. Partridge, L. C. Wrobel, An inverse geometry problem for the localisation of skin tumours by thermal analysis, Engineering Analysis with Boundary Elements 31 (10) (2007) 803-811.

[35] J. M. Luna, R. Romero-Mendez, A. Hernandez-Guerrero, F. Elizalde-Blancas, Procedure to estimate thermophysical and geometrical parameters of embedded cancerous lesions using thermography, Journal of Biomechanical Engineering 134 (3) (2012) 031008 .

[36] J. M. Luna, A. Hernández Guerrero, R. Romero Méndez, J. L. Luviano Ortiz, Solution of the inverse bio-heat transfer problem for a simplified dermatological application: Case of skin cancer, Ingeniería Mecánica, Tecnología y Desarrollo 4 (6) (2014) 219-228.

[37] F. R. Bueno, P. W. Partridge, An inverse model for locating skin tumours in 3D using the genetic algorithm with the dual reciprocity boundary element method, Latin American Journal of Solids and Structures 10 (6) (2013) 1061-1079. 
[38] S. Hossain, F. Mohammadi, M. Abdelaal, Localization and parameter estimation of tumor by thermography, in: Electrical and Computer Engineering (CCECE), 2014 IEEE 27th Canadian Conference, IEEE, 2014, pp. 1-5.

[39] A. Bhowmik, R. Repaka, Estimation of growth features and thermophysical properties of melanoma within 3-D human skin using genetic algorithm and simulated annealing, International Journal of Heat and Mass Transfer 98 (2016) 81-95.

[40] J. Iljaž, L. Wrobel, M. Hriberšek, J. Marn, Subdomain BEM formulations for the solution of bio-heat problems in biological tissue with melanoma lesions, Engineering Analysis with Boundary Elements 83 (2017) 25-42.

[41] A. Bhowmik, R. Repaka, S. C. Mishra, Thermographic evaluation of early melanoma within the vascularized skin using combined non-Newtonian blood flow and bioheat models, Computers in Biology and Medicine 53 (2014) 206-219.

[42] H. H. Pennes, Analysis of tissue and arterial blood temperatures in the resting human forearm, Journal of Applied Physiology 1 (2) (1948) 93-122.

[43] M. P. Çetingül, C. Herman, A heat transfer model of skin tissue for the detection of lesions: sensitivity analysis, Physics in Medicine and Biology 55 (19) (2010) 59335951.

[44] E. Burnaev, I. Panin, B. Sudret, Efficient design of experiments for sensitivity analysis based on polynomial chaos expansions, Annals of Mathematics and Artificial Intelligence (2017) 1-21.

[45] J.-H. Kim, H.-C. Lee, J.-H. Kim, Y.-S. Choi, J.-Y. Yoon, I.-S. Yoo, W.-C. Choi, Improvement of hydrodynamic performance of a multiphase pump using design of experiment techniques, Journal of Fluids Engineering 137 (8) (2015) 08130-1-15.

[46] J. Dhariwal, R. Banerjee, An approach for building design optimization using design of experiments, in: Building Simulation, Vol. 10, Springer, 2017, pp. 323-336.

[47] J. P. N. Kumar, S. J. Kumar, R. S. Jeyathilak, M. Venkatesh, A. S. Christopher, K. Ganesh, Effect of design parameters on the static mechanical behaviour of metal bellows using design of experiment and finite element analysis, International Journal on Interactive Design and Manufacturing (IJIDeM) 11 (3) (2017) 535-545.

[48] C.-K. Kim, J.-Y. Yoon, Optimal design of groove shape on passive micromixer using design of experiment technique, Proceedings of the Institution of Mechanical Engineers, Part E: Journal of Process Mechanical Engineering 231 (4) (2017) 880-887. 